\title{
Polyunsaturated Fatty Acids and Risk of Ischemic Stroke
}

\author{
Stine Krogh Venø ${ }^{1,2}$, Erik Berg Schmidt ${ }^{1,2}$ and Christian Sørensen Bork 1,2,* \\ 1 Department of Cardiology, Aalborg University Hospital, 9000 Aalborg, Denmark \\ 2 Department of Clinical Medicine, Aalborg University, 9000 Aalborg, Denmark \\ * Correspondence: c.bork@rn.dk; Tel.: +45-97666899
}

Received: 3 June 2019; Accepted: 25 June 2019; Published: 27 June 2019

\begin{abstract}
Ischemic stroke is a major cause of death and morbidity worldwide. It has been suggested that polyunsaturated fatty acids (PUFAs) may be associated with a lower risk ischemic stroke, but this has been far less studied than their role for coronary heart disease. In this paper, we summarize the main findings from previous follow-up studies investigating associations between intake or biomarkers of the major PUFAs including alpha-linolenic acid (ALA), marine n-3 PUFAs and linoleic acid (LA) and the development of ischemic stroke. Several follow-up studies have suggested that marine n-3 PUFAs may be associated with a lower risk of ischemic stroke although results have not been consistent and limited knowledge exist on the individual marine n-3 PUFAs and ischemic stroke and its subtypes. The role of ALA is less clear, but most studies have not supported that ALA is appreciably associated with ischemic stroke risk. Some studies have supported that LA might be associated with a lower risk of total ischemic stroke, while limited evidence exist on PUFAs and ischemic stroke subtypes. The associations may depend on the macronutrients that PUFAs replace and this substitution aspect together with focus on dietary patterns represent interesting areas for future research.
\end{abstract}

Keywords: polyunsaturated fatty acids; ischemic stroke; observational prospective studies; biomarkers

\section{Introduction}

Ischemic stroke is characterized by an episode of neurological dysfunction attributed to cerebral infarction in the perfusion territory of an stenosed or occluded artery [1]. Ischemic stroke is a major cause of death and disability worldwide, but some studies have indicated that adherence to a healthy diet and lifestyle may be of importance for reduction of ischemic stroke risk [2,3].

An important part of a healthy diet is believed to be an increased intake of polyunsaturated fatty acids (PUFAs). PUFAs are organic acids that naturally contains two or more double bonds in their carbon chain and are named according to the number, configuration and position of these double bonds [4]. PUFAs are upon ingestion incorporated into cell membranes and storage pools, utilised in energy production or converted into longer and more unsaturated fatty acids, which may give rise to biological compounds that may affect a variety of biological processes [5]. PUFAs have traditionally been divided into n-3 PUFAs and n-6 PUFAs and these two families each consist of fatty acids with varying carbon chain length and degree of unsaturation, which are important for their physiological and biological properties [4]. The major n-3 PUFAs include the short-chain plant-derived n-3 PUFA, alpha-linolenic acid (ALA, 18:3n-3), and the longer-chain marine n-3 PUFAs eicosapentaenoic acid (EPA, 20:5n-3), docosapentaenoic acid (DPA, 22:5n-3) and docosahexaenoic acid (DHA, 22:6n-3).

The major dietary n-6 PUFA is linoleic acid (LA, 18:2n-6) and together with ALA are the PUFAs consumed in largest amounts. The typical intake of LA in Western populations range between 10 to 
$17 \mathrm{~g} / \mathrm{d}$, while the average intake of ALA varies between 0.5 to $2.3 \mathrm{~g} / \mathrm{d}$ [5-7]. The major sources of LA include plant oils, meat and eggs [6], while ALA is found in large amounts in some plant oils, but can be also be found in walnuts, green vegetables, whole-grain cereals, margarines, dairy products and meat [8-10]. LA and ALA are precursors for further desaturation and elongation products along a shared metabolic pathway. LA can thus be converted into arachidonic acid (AA, 20:4n-6), while ALA can be converted into LC n-3 PUFAs, however, the conversion efficiency seems to be limited in humans and influenced by gender, genetics and intake of other fatty acids $[5,11]$. The LC n-3 PUFAs can be acquired from seafood and especially fatty fish and their biological effects has been extensively studied over the recent decades since Dyerberg and colleagues hypothesized that LC n-3 PUFAs in particular EPA were protective against atherothrombosis [12-14].

LC n-3 PUFAs has been ascribed several effects that might be beneficial in the prevention of ischemic stroke including lowering of triglycerides, inflammation, blood pressure and platelet aggregability, and improved endothelial function and atherosclerotic plaque stability, which likely are mediated by a combination of mechanisms [4,14-17]. On the other hand the biological effects of other PUFAs have been less studied, but few studies have suggested that ALA might be associated with anti-inflammatory and anti-atherosclerotic properties [18]. Whether these suggested effects is mediated by ALA per se or can be attributed to its role as a precursor for LC n-3 PUFAs, however, remains unclear. LA has been reported to be associated with lowering of low-density lipoprotein (LDL) cholesterol, blood pressure and insulin resistance [19], all important for atherosclerotic risk. Concerns have, however, been raised that LA could confer a higher risk of vascular disease due to its role as a precursor of AA that gives rise to eicosanoids considered pro-inflammatory and pro-thrombotic including formation of proinflammatory leukotriene B4 from leukocytes and proaggregatory and vasoconstrictive thromboxane A2 [4]. However, stable isotope studies and clinical trials have indicated that high intakes of LA do not seem to significantly increase tissue levels of AA or increased production of inflammatory mediators [20]. Indeed, there is actually some indications that LA might possess anti-inflammatory effects [20,21].

Several studies have suggested that LC n-3 PUFAs and LA may be associated with a lower risk of coronary heart disease (CHD) [4,22-24]. Although, CHD and ischemic stroke may share pathophysiological similarities, less is known about the role of PUFAs in relation to risk of ischemic stroke and previous studies have not yielded consistent results.

The objective of this paper was to summarize the main findings from previous follow-up studies investigating associations between intake or biomarkers of exposure of the major PUFAs (ALA, LC n-3 PUFAs, and LA) and ischemic stroke.

\section{ALA and Ischemic Stroke}

Few studies have investigated the association between ALA intake and ischemic stroke [25-29] (Table 1). In the Monitoring Project on Risk Factors for Chronic Diseases Study (MORGEN Study), dietary intake of ALA was inversely associated with the rate of total ischemic stroke among Dutch men and women [25]. In the Cardiovascular Health Study (CHS), indications of a lower rate of total ischemic stroke were observed when comparing the highest quintile of ALA intake with the lowest, but no consistent pattern of association was observed across quintiles of ALA in this cohort of US men and women [26]. Further, no clear association was observed between ALA intake and the rate of total ischemic stroke among women enrolled into the Women's Health Study [27] or the Swedish Mammography Cohort [28] and among men and women enrolled into the Diet, Cancer and Health $(\mathrm{DCH})$ cohort [29]. Limited knowledge is available regarding the associations between ALA intake and the risk of ischemic stroke subtypes but no appreciable pattern of associations were observed between intake of ALA and the rate of ischemic stroke subtypes caused by large artery atherosclerosis, small vessel-occlusion or cardioembolism in the DCH cohort [29] (Table 2). 
Table 1. Characteristics of observational follow-up studies investigating intake of alpha-linolenic acid (ALA) and total ischemic stroke.

\begin{tabular}{|c|c|c|c|c|c|c|c|c|c|c|}
\hline $\begin{array}{l}\text { Author and } \\
\text { Study }\end{array}$ & $\begin{array}{l}\text { Study Population } \\
\text { and Country }\end{array}$ & Sex & $\begin{array}{c}\text { No. of } \\
\text { Participants }\end{array}$ & $\begin{array}{l}\text { Baseline } \\
\text { Age (yrs) }\end{array}$ & $\begin{array}{c}\text { Exposure } \\
\text { Assessment }\end{array}$ & Intake (g/d) & $\begin{array}{l}\text { No. of } \\
\text { Cases }\end{array}$ & $\begin{array}{c}\text { Follow-up } \\
\text { (yrs) }\end{array}$ & $\begin{array}{l}\text { Hazard Ratios } \\
(95 \% \mathrm{CI})\end{array}$ & Variables Controlled for \\
\hline $\begin{array}{l}\text { De Goede et } \\
\text { al. } 2011 \text { [25] }\end{array}$ & $\begin{array}{l}\text { The MORGEN } \\
\text { Study cohort; The } \\
\text { Netherlands }\end{array}$ & $\mathrm{M}+\mathrm{W}$ & 19,896 & $20-65$ & FFQ & $\begin{array}{l}\text { Q1: } 1.0 \\
\text { Q3: } 1.3 \\
\text { Q5: } 1.9\end{array}$ & 144 & 10.5 & $\begin{array}{l}\text { Q2: } 0.63(0.39 ; 1.02) \\
\text { Q3: } 0.45(0.26 ; 0.77) \\
\text { Q4: } 0.55(0.33 ; 0.92) \\
\text { Q5: } 0.70(0.43 ; 1.12) \\
\end{array}$ & $\begin{array}{c}\text { Age; sex; BMI; total energy intake; } \\
\text { cigarette smoking; educational } \\
\text { level; parental history of MI; } \\
\text { alcohol. }\end{array}$ \\
\hline $\begin{array}{l}\text { Larsson et al. } \\
2012 \text { [28] }\end{array}$ & $\begin{array}{l}\text { The Swedish } \\
\text { Mammography } \\
\text { Cohort; Sweden }\end{array}$ & W & 34,670 & 49-83 & FFQ & $\begin{array}{l}\text { Q1: } 0.9 \\
\text { Q3: } 1.1 \\
\text { Q5: } 1.5\end{array}$ & 1310 & 10.4 & $\begin{array}{l}\text { Q2: } 0.99(0.83 ; 1.17) \\
\text { Q3: } 1.03(0.86 ; 1.22) \\
\text { Q4: } 1.00(0.84 ; 1.19) \\
\text { Q5: } 1.11(0.93 ; 1.32)\end{array}$ & $\begin{array}{l}\text { Age; smoking status and } \\
\text { pack-years of smoking; education; } \\
\text { BMI; physical activity; } \\
\text { hypertension; diabetes; aspirin; } \\
\text { family history of MI; intakes of } \\
\text { alcohol, protein, fiber and } \\
\text { cholesterol. }\end{array}$ \\
\hline $\begin{array}{l}\text { Fretts et al. } \\
2014 \text { [26] }\end{array}$ & $\begin{array}{l}\text { The CHS cohort; } \\
\text { United States }\end{array}$ & $\mathrm{M}+\mathrm{W}$ & 2583 & $\geq 65$ & FFQ & $\begin{array}{l}\text { Q1: } 1.3 \%^{1} \\
\text { Q3: } 1.8 \%^{1} \\
\text { Q5: } 2.4 \%^{1}\end{array}$ & 278 & $9.6^{2}$ & $\begin{array}{l}\text { Q2: } 0.89(0.61 ; 1.30) \\
\text { Q3: } 0.84(0.58 ; 1.22) \\
\text { Q4: } 1.08(0.75 ; 1.54) \\
\text { Q5: } 0.70(0.47 ; 1.04)\end{array}$ & $\begin{array}{l}\text { Age; sex; total energy intake; race; } \\
\text { enrollment site; education; } \\
\text { smoking status; diabetes; BMI; } \\
\text { waist circumference; physical } \\
\text { activity; alcohol; treated } \\
\text { hypertension. } \\
\end{array}$ \\
\hline $\begin{array}{l}\text { Rhee et al. } \\
2017 \text { [27] }\end{array}$ & $\begin{array}{l}\text { The WHS cohort; } \\
\text { United States }\end{array}$ & W & 38,392 & $\geq 45$ & FFQ & $\begin{array}{l}\text { Q1: } 0.7 \%^{3} \\
\text { Q3: } 1.0 \%^{3} \\
\text { Q5: } 1.4 \%^{3}\end{array}$ & 807 & $18.2^{2}$ & $\begin{array}{l}\text { Q2: } 0.89(0.71 ; 1.13) \\
\text { Q3: } 0.89(0.71 ; 1.12) \\
\text { Q4: } 1.05(0.84 ; 1.30) \\
\text { Q5: } 1.02(0.82 ; 1.27)\end{array}$ & $\begin{array}{l}\text { Age; randomized treatment } \\
\text { assignment; BMI; smoking; alcohol; } \\
\text { physical activity; oral } \\
\text { contraceptive use; HRT; } \\
\text { multivitamins; total energy intake; } \\
\text { family history of MI; hypertension; } \\
\text { high cholesterol; diabetes. }\end{array}$ \\
\hline $\begin{array}{l}\text { Bork et al. } \\
2018 \text { [29] }\end{array}$ & $\begin{array}{l}\text { The DCH cohort; } \\
\text { Denmark }\end{array}$ & $\mathrm{M}+\mathrm{W}$ & 55,018 & $50-65$ & FFQ & $\begin{array}{l}\text { Q1: } 1.2 \\
\text { Q3: } 1.8 \\
\text { Q5: } 2.6\end{array}$ & 1859 & 13.5 & $\begin{array}{l}\text { Q2: } 1.03(0.87 ; 1.21) \\
\text { Q3: } 1.12(0.95 ; 1.31) \\
\text { Q4: } 1.03(0.87 ; 1.22) \\
\text { Q5: } 1.10(0.93 ; 1.31)\end{array}$ & $\begin{array}{l}\text { Age; gender; length of schooling; } \\
\text { smoking; physical activity; waist } \\
\text { circumference adjusted for BMI; } \\
\text { alcohol. }\end{array}$ \\
\hline
\end{tabular}

Abbreviations: MORGEN, The Monitoring Project on Risk Factors for Chronic Diseases Study; M, men; W, Women; FFQ, Food Frequency Questionnaire; Q, Quintile; CI, Confidence interval; BMI, Body mass index; MI, Myocardial infarction; CHS, Cardiovascular Health Study; WHS, Women's Health Study; HRT, Hormone replacement therapy; DCH, Diet, Cancer and Health. The presented measures of association was selected for models that included adjustment for lifestyle factors without including dietary covariates whenever possible. The lowest quintile of intake was used as reference. ${ }^{1}$ Percentage of total fatty acids; ${ }^{2}$ Follow-up time was calculated as person-years of follow-up divided by the total number of participants; ${ }^{3}$ Percentage of total energy intake. 
Table 2. Measures of association obtained in observational follow-up and nested case-control studies investigating intake or biomarkers of major PUFAs in relation to ischemic stroke subtypes.

\begin{tabular}{|c|c|c|c|}
\hline $\begin{array}{c}\text { Author and Exposures of } \\
\text { Interest }\end{array}$ & $\begin{array}{l}\text { Large Artery Occlusive } \\
\text { Infarctions }\end{array}$ & $\begin{array}{l}\text { Small-Vessel and Lacunar } \\
\text { Infarctions }\end{array}$ & Cardio-Embolic Infarctions \\
\hline \multicolumn{4}{|l|}{ Iso et al. 2001 [30] } \\
\hline \multirow[t]{2}{*}{ No. cases } & 88 & 142 & \\
\hline & HR Q2-5 vs. Q1: & HR Q2-5 vs. Q1: & \\
\hline \multirow{4}{*}{ EPA+DHA intake } & Q2: $0.56(0.28 ; 1.10)$ & Q2: $0.89(0.55 ; 1.43)$ & \\
\hline & Q3: $0.50(0.25 ; 1.02)$ & Q3: $0.59(0.34 ; 1.00)$ & \\
\hline & Q4: $0.96(0.51 ; 1.81)$ & Q4: $0.67(0.39 ; 1.14)$ & \\
\hline & Q5: $1.09(0.52 ; 2.29)$ & Q5: $0.37(0.19 ; 0.73)$ & \\
\hline \multicolumn{4}{|l|}{ Iso et al. 2002 [31] } \\
\hline No. of cases & 19 & 95 & 8 \\
\hline ALA in serum & $\begin{array}{c}\text { OR: } 0.82 \text { (NS, CI not re-ported) } \\
\text { per } 1 \text { SD increase }{ }^{1}\end{array}$ & $\begin{array}{c}\text { OR: } 0.82 \text { (NS, CI not re-ported) } \\
\text { per } 1 \mathrm{SD} \text { increase }{ }^{1}\end{array}$ & $\begin{array}{c}\text { OR: } 0.89 \text { (NS, CI not re-ported) } \\
\text { per } 1 \text { SD increase }{ }^{1}\end{array}$ \\
\hline EPA in serum & $\begin{array}{c}\text { OR: } 2.28(1.14 ; 4.57) \text { per } 1 \mathrm{SD} \\
\text { increase }^{1}\end{array}$ & $\begin{array}{c}\text { OR: } 0.89 \text { (NS, CI not re-ported) } \\
\text { per } 1 \text { SD increase }{ }^{1}\end{array}$ & $\begin{array}{c}\text { OR: } 0.63 \text { (NS, CI not re-ported) } \\
\text { per } 1 \mathrm{SD} \text { increase }{ }^{1}\end{array}$ \\
\hline DPA in serum & $\begin{array}{c}\text { OR: } 1.35 \text { (NS, CI not re-ported) } \\
\text { per } 1 \text { SD increase }{ }^{1}\end{array}$ & $\begin{array}{c}\text { OR: } 1.17 \text { (NS, CI not re-ported) } \\
\text { per } 1 \text { SD increase }{ }^{1}\end{array}$ & $\begin{array}{c}\text { OR: } 1.59 \text { (NS, CI not re-ported) } \\
\text { per } 1 \mathrm{SD} \text { increase }{ }^{1}\end{array}$ \\
\hline DHA in serum & $\begin{array}{c}\text { OR: } 1.53 \text { (NS, CI not re-ported) } \\
\text { per } 1 \text { SD increase }{ }^{1}\end{array}$ & $\begin{array}{c}\text { OR: } 1.02 \text { (NS, CI not re-ported) } \\
\text { per } 1 \text { SD increase }{ }^{1}\end{array}$ & $\begin{array}{c}\text { OR: } 1.06 \text { (NS, CI not re-ported) } \\
\text { per } 1 \text { SD increase }{ }^{1}\end{array}$ \\
\hline LA in serum & $\begin{array}{c}\text { OR: } 0.66 \text { (NS, CI not re-ported) } \\
\text { per } 1 \text { SD increase }{ }^{1}\end{array}$ & $\begin{array}{c}\text { OR: } 0.63(0.46 ; 0.88) \text { per } 1 \mathrm{SD} \\
\text { increase }\end{array}$ & $\begin{array}{c}\text { OR: } 0.92 \text { (NS, CI not re-ported) } \\
\text { per } 1 \text { SD increase }{ }^{1}\end{array}$ \\
\hline \multicolumn{4}{|l|}{ Yaemsiri et al. 2013 [32] } \\
\hline No. of cases & 96 & 250 & 209 \\
\hline EPA in serum & $\begin{array}{c}\text { OR: } 0.51(0.23 ; 1.14) \text { per } 1 \mathrm{SD} \\
\text { increase }^{2}\end{array}$ & $\begin{array}{c}\text { OR: } 0.58(0.36 ; 0.94) \text { per } 1 \mathrm{SD} \\
\text { increase }^{2}\end{array}$ & $\begin{array}{c}\text { OR: } 0.84(0.55 ; 1.30) \text { per } 1 \mathrm{SD} \\
\text { increase }^{2}\end{array}$ \\
\hline DPA in serum & $\begin{array}{c}\text { OR: } 0.83(0.47 ; 1.47) \text { per } 1 \mathrm{SD} \\
\text { increase }^{2}\end{array}$ & $\begin{array}{c}\text { OR: } 0.64(0.44 ; 0.94) \text { per } 1 \mathrm{SD} \\
\text { increase }^{2}\end{array}$ & $\begin{array}{c}\text { OR: } 0.82(0.55 ; 1.24) \text { per } 1 \mathrm{SD} \\
\text { increase }^{2}\end{array}$ \\
\hline DHA in serum & $\begin{array}{c}\text { OR: } 0.70(0.36 ; 1.36) \text { per } 1 \mathrm{SD} \\
\text { increase }^{2}\end{array}$ & $\begin{array}{c}\text { OR: } 0.61(0.40 ; 0.93) \text { per } 1 \mathrm{SD} \\
\text { increase }^{2}\end{array}$ & $\begin{array}{c}\text { OR: } 0.66(0.43 ; 1.02) \text { per } 1 \mathrm{SD} \\
\text { increase }^{2}\end{array}$ \\
\hline \multicolumn{4}{|l|}{ Bork et al. 2018 [29] } \\
\hline \multirow[t]{2}{*}{ No. of cases } & 316 & 835 & 102 \\
\hline & HR Q2-5 vs. Q1: & HR Q2-5 vs. Q1: & HR Q2-5 vs. Q1: \\
\hline \multirow{4}{*}{ ALA intake } & Q2: $1.03(0.71 ; 1.51)$ & Q2: $1.08(0.84 ; 1.39)$ & Q2: $1.30(0.62 ; 2.72)$ \\
\hline & Q3: $0.91(0.62 ; 1.34)$ & Q3: $1.23(0.97 ; 1.56)$ & Q3: $1.23(0.59 ; 2.56)$ \\
\hline & Q4: $0.90(0.61 ; 1.34)$ & Q4: $1.09(0.84 ; 1.40)$ & Q4: $1.08(0.51 ; 2.29)$ \\
\hline & Q5: $0.96(0.65 ; 1.44)$ & Q5: $1.25(0.98 ; 1.61)$ & Q5: $1.31(0.63 ; 2.76)$ \\
\hline \multicolumn{4}{|l|}{ Bork et al. 2018 [33] } \\
\hline \multirow[t]{2}{*}{ No. of cases } & 297 & 772 & 99 \\
\hline & HR Q2-5 vs. Q1: & HR Q2-5 vs. Q1: & HR Q2-5 vs. Q1: \\
\hline \multirow{4}{*}{ ALA in adipose tissue } & Q2: $0.72(0.48 ; 1.08)$ & Q2: $1.00(0.77 ; 1.31)$ & Q2: $1.05(0.53 ; 2.06)$ \\
\hline & Q3: $0.63(0.41 ; 0.96)$ & Q3: $0.96(0.73 ; 1.25)$ & Q3: $1.16(0.59 ; 2.27)$ \\
\hline & Q4: $0.83(0.56 ; 1.22)$ & Q4: $1.02(0.78 ; 1.33)$ & Q4: $0.90(0.43 ; 1.87)$ \\
\hline & Q5: $0.95(0.65 ; 1.40)$ & Q5: $1.05(0.80 ; 1.38)$ & Q5: $1.91(0.98 ; 3.70)$ \\
\hline \multicolumn{4}{|l|}{ Saber et al. 2017 [34] } \\
\hline No. of cases & \multicolumn{2}{|c|}{408} & 256 \\
\hline \multirow{4}{*}{ EPA in PL } & \multicolumn{2}{|c|}{ HR Q2-4 vs. Q1: } & HR Q2-4 vs. Q1: \\
\hline & \multicolumn{2}{|c|}{$\mathrm{Q} 2: 1.03(0.70 ; 1.52)$} & Q2: $1.10(0.72 ; 1.66)$ \\
\hline & \multicolumn{2}{|c|}{ Q3: $0.97(0.65 ; 1.44)$} & Q3: $0.96(0.62 ; 1.50)$ \\
\hline & \multicolumn{2}{|c|}{ Q4: $1.06(0.71 ; 1.59)$} & Q4: $0.94(0.61 ; 1.45)$ \\
\hline \multirow{4}{*}{ DPA in PL } & \multirow{4}{*}{\multicolumn{2}{|c|}{$\begin{array}{l}\text { HR for Q2-4 vs. Q1: } \\
\text { Q2: } 1.05(0.72 ; 1.53) \\
\text { Q3: } 1.02(0.69 ; 1.53) \\
\text { Q4: } 0.98(0.64 ; 1.53)\end{array}$}} & HR Q2-4 vs. Q1: \\
\hline & & & Q2: $0.75(0.50 ; 1.14)$ \\
\hline & & & Q3: $0.83(0.55 ; 1.35)$ \\
\hline & & & Q4: $0.59(0.38 ; 0.93)$ \\
\hline \multirow{4}{*}{ DHA in PL } & \multicolumn{2}{|c|}{ HR Q2-4 vs. Q1: } & HR Q2-4 vs. Q1: \\
\hline & \multicolumn{2}{|c|}{ Q2: $0.88(0.60 ; \tilde{1.16})$} & Q2: $1.25(0.83 ; 1.90)$ \\
\hline & Q3: 0.72 & $0.48 ; 1.07)$ & Q3: $1.07(0.69 ; 1.65)$ \\
\hline & Q4: 0.53 & $0.34 ; 0.83)$ & Q4: $1.09(0.69 ; 1.72)$ \\
\hline Venø et al. 2019 [35] & & & \\
\hline
\end{tabular}


Table 2. Cont

\begin{tabular}{|c|c|c|c|}
\hline $\begin{array}{c}\text { Author and Exposures of } \\
\text { Interest }\end{array}$ & $\begin{array}{l}\text { Large Artery Occlusive } \\
\text { Infarctions }\end{array}$ & $\begin{array}{l}\text { Small-Vessel and Lacunar } \\
\text { Infarctions }\end{array}$ & Cardio-Embolic Infarctions \\
\hline $\begin{array}{c}\text { No. of cases in dietary } \\
\text { analyses }\end{array}$ & 319 & 844 & 102 \\
\hline No. of cases in AT analyses & 300 & 781 & 99 \\
\hline \multirow{4}{*}{ Total LC n-3 intake } & HR Q2-4 vs. Q1: & HR Q2-4 vs. Q1: & HR Q2-4 vs. Q1: \\
\hline & Q2: $0.97(0.72 ; \hat{1.30})$ & $\mathrm{Q} 2: 1.15(0.94 ; \hat{1.40})$ & Q2: $1.36(0.69 ; 2.66)$ \\
\hline & Q3: $0.88(0.65 ; 1.19)$ & Q3: $1.20(0.98 ; 1.45)$ & Q3: $1.49(0.78 ; 2.88)$ \\
\hline & Q4: $0.69(0.50 ; 0.95)$ & Q4: $1.06(0.87: 1.30)$ & Q4: $2.50(1.38 ; 4.53)$ \\
\hline \multirow{4}{*}{ Total LC n-3 in AT } & HR Q2-4 vs. Q1: & HR Q2-4 vs. Q1: & HR Q2-4 vs. Q1: \\
\hline & Q2: $0.86(0.61 ; 1.22)$ & Q2: $0.91(0.72 ; 1.15)$ & Q2: $2.08(1.04 ; 4.15)$ \\
\hline & Q3: $1.09(0.78 ; 1.52)$ & Q3: $1.05(0.83 ; 1.32)$ & Q3: $2.04(1.03 ; 4.04)$ \\
\hline & Q4: $0.78(0.53 ; 1.13)$ & Q4: $0.99(0.79 ; 1.26)$ & Q4: $2.63(1.33 ; 5.19)$ \\
\hline \multirow{4}{*}{ EPA intake } & HR Q2-4 vs. Q1: & HR Q2-4 vs. Q1: & HR Q2-4 vs. Q1: \\
\hline & Q2: $0.86(0.64 ; 1.16)$ & Q2: $1.14(0.94 ; 1.38)$ & Q2: $1.17(0.58 ; 2.35)$ \\
\hline & Q3: $0.82(0.60 ; 1.11)$ & Q3: $1.16(0.96 ; 1.41)$ & Q3: $2.34(1.27 ; 4.30)$ \\
\hline & Q4: $0.66(0.48 ; 0.91)$ & Q4: $1.05(0.86 ; 1.28)$ & Q4: $2.02(1.09 ; 3.73)$ \\
\hline \multirow{4}{*}{$\mathrm{EPA}$ in AT } & HR Q2-4 vs. Q1: & HR Q2-4 vs. Q1: & HR Q2-4 vs. Q1: \\
\hline & Q2: $0.96(0.70 ; 1.32)$ & Q2: $0.84(0.68 ; 1.04)$ & Q2: $1.13(0.61 ; 2.11)$ \\
\hline & Q3: $0.64(0.43 ; 0.94)$ & Q3: $0.61(0.47 ; 0.79)$ & Q3: $1.06(0.52 ; 2.14)$ \\
\hline & Q4: $0.52(0.36 ; 0.76)$ & Q4: $0.69(0.55 ; 0.88)$ & $\mathrm{Q} 4: 1.52(0.82 ; 2.81)$ \\
\hline \multirow{4}{*}{ DPA intake } & HR Q2-4 vs. Q1: & HR Q2-4 vs. Q1: & HR Q2-4 vs. Q1: \\
\hline & Q2: $1.01(0.74 ; 1.38)$ & Q2: $1.27(1.04 ; 1.55)$ & Q2: 0.80 (0.41: 1.56$)$ \\
\hline & Q3: $0.91(0.67 ; 1.25)$ & Q3: $1.21(0.99 ; 1.48)$ & Q3: $1.14(0.63 ; 2.08)$ \\
\hline & Q4: $0.88(0.64 ; 1.21)$ & Q4: $1.19(0.98 ; 1.46)$ & Q4: $1.68(0.96 ; 2.91)$ \\
\hline \multirow{4}{*}{ DPA in AT } & HR Q2-4 vs. Q1: & HR Q2-4 vs. Q1: & HR Q2-4 vs. Q1: \\
\hline & Q2: $1.46(1.04 ; 2.07)$ & Q2: $1.37(1.08 ; 1.74)$ & Q2: $2.45(1.19 ; 5.05)$ \\
\hline & Q3: $1.23(0.86 ; 1.76)$ & Q3: $1.29(1.01 ; 1.64)$ & Q3: $2.61(1.30 ; 5.25)$ \\
\hline & Q4: $1.02(0.69 ; 1.51)$ & Q4: $1.19(0.93 ; 1.52)$ & $\mathrm{Q} 4: 3.06(1.50 ; 6.24)$ \\
\hline \multirow{4}{*}{ DHA intake } & HR Q2-4 vs. Q1: & HR Q2-4 vs. Q1: & HR Q2-4 vs. Q1: \\
\hline & Q2: $0 . \overline{90}(0.67 ; \hat{1.22})$ & $\mathrm{Q} 2: 1.26(1.04 ; \hat{1.54})$ & Q2: $0.97(0.50 ; \hat{1.89})$ \\
\hline & Q3: $0.86(0.63 ; 1.16)$ & Q3: $1.17(0.96 ; 1.43)$ & Q3: $1.27(0.68 ; 2.36)$ \\
\hline & Q4: $0.72(0.53 ; 0.99)$ & Q4: $1.13(0.93 ; 1.38)$ & Q4: $2.12(1.21 ; 3.69)$ \\
\hline \multirow{4}{*}{ DHA in AT } & HR Q2-4 vs. Q1: & HR Q2-4 vs. Q1: & HR Q2-4 vs. Q1: \\
\hline & Q2: $0.94(0.67 ; 1.32)$ & Q2: $0.86(0.68 ; 1.09)$ & Q2: $1.37(0.71 ; 2.64)$ \\
\hline & Q3: $1.08(0.77 ; 1.52)$ & Q3: $1.07(0.85 ; 1.34)$ & Q3: $1.64(0.87 ; 3.10)$ \\
\hline & Q4: $0.79(0.54 ; 1.16)$ & Q4: $0.92(0.72 ; 1.17)$ & Q4: $2.00(1.04 ; 3.84)$ \\
\hline \multicolumn{4}{|l|}{ Venø et al. 2017 [36] } \\
\hline \multirow{8}{*}{ LA intake } & 319 & 844 & 102 \\
\hline & LA for SFA: & LA for SFA: & LA for SFA: \\
\hline & HR: $0.84(0.57 ; 1.25)$ & HR: $0.96(0.75 ; 1.23)$ & HR: $1.46(0.75 ; 2.85)$ \\
\hline & LA for MUFA: & LA for MUFA: & LA for MUFA: \\
\hline & HR: $1.05(0.58 ; 1.90)$ & HR: $0.67(0.46 ; 0.96)$ & HR: $1.35(0.51 ; 3.55)$ \\
\hline & LA for glycemic & LA for glycemic & LA for glycemic \\
\hline & carbohydrates: & carbohydrates: & carbohydrates: \\
\hline & HR: $0.96(0.64 ; 1.44)$ & HR: $0.82(0.64 ; 1.05)$ & HR: $1.55(0.81 ; 3.00)$ \\
\hline \multicolumn{4}{|l|}{ Venø et al. 2018 [37] } \\
\hline No. of cases & 300 & 781 & 99 \\
\hline \multirow{4}{*}{ LA in AT } & HR Q2-4 vs. Q1: & HR Q2-4 vs. Q1: & HR Q2-4 vs. Q1: \\
\hline & Q2: $0.72(0.51 ; 1.01)$ & Q2: $0.90(0.72 ; .13)$ & Q2: $1.28(0.75 ; 2.19)$ \\
\hline & Q3: $0.84(0.61 ; 1.17)$ & Q3: $0.87(0.69 ; 1.03)$ & Q3: $0.71(0.37 ; 1.37)$ \\
\hline & Q4: $0.61(0.43 ; 0.88)$ & Q4: $0.87(0.69 ; 1.11)$ & Q4: $0.86(0.46 ; 1.59)$ \\
\hline
\end{tabular}

Abbreviations: NS, Not statistically significant; AT, Adipose tissue. ${ }^{1}$ Univariate analysis. ${ }^{2} 99 \%$ Confidence interval. The presented measures of association was selected for models that included adjustment for lifestyle factors without including dietary covariates whenever possible. See Table 1 and Tables 3-7 for study characteristics.

The findings from previous follow-up and nested-case control studies using biomarkers of ALA to investigate associations with ischemic stroke have not been conclusive [26,31-33,38-40] (Table 3). In a large case-cohort study based on data from the DCH cohort we reported indications of a U-shaped pattern of association between adipose tissue content of ALA and the rate of total ischemic stroke although not statistically significant [33]. A follow-up study based on the Finish Kuopio Ischaemic Heart Disease Risk Factors (KIHD) study also reported indications of a U-shaped pattern of association between quartiles of ALA content in serum and total ischemic stroke, while a nested case-control study reported a modest non-significant inverse association between ALA in serum and total ischemic stroke [32,40]. In contrast, several other biomarker studies did not find ALA content in serum, plasma phospholipids or cholesterol esters associated with the risk of total ischemic stroke $[26,31,38,39]$. 
Table 3. Characteristics of observational follow-up and nested case-control studies investigating biomarkers of alpha-linolenic acid (ALA) and total ischemic stroke.

\begin{tabular}{|c|c|c|c|c|c|c|c|c|c|}
\hline $\begin{array}{l}\text { Author and } \\
\text { Study }\end{array}$ & $\begin{array}{l}\text { Study Population, } \\
\text { Design and Country }\end{array}$ & Sex & $\begin{array}{l}\text { No. of Partici-Pants } \\
\text { and Cases }\end{array}$ & $\begin{array}{c}\text { Baseline Age } \\
\text { (yrs) }\end{array}$ & Biomarker & $\begin{array}{c}\text { Concentrations } \\
(\%)\end{array}$ & $\begin{array}{c}\text { Follow-Up } \\
(\mathrm{yrs})\end{array}$ & $\begin{array}{c}\text { Measure of } \\
\text { Association }(95 \% \mathrm{CI})\end{array}$ & Variables Controlled for \\
\hline $\begin{array}{c}\text { Iso et al. } 2002 \\
\text { [31] }\end{array}$ & $\begin{array}{c}\text { A Japanese } \\
\text { cardiovascular risk } \\
\text { survey population; } \\
\text { Nested case-control; } \\
\text { Japan } \\
\end{array}$ & $\mathrm{M}+\mathrm{W}$ & $\begin{array}{l}122 \text { cases \& } 366 \\
\text { controls }\end{array}$ & $40-85$ & ALA in serum & $\begin{array}{c}\text { Cases: } 1.0 \% \\
\text { Controls: } 1.0 \%\end{array}$ & Not reported & $\begin{array}{l}\text { OR: } 0.86 \text { (NS, CI not } \\
\text { reported) per 1 SD } \\
\text { increase in ALA }\end{array}$ & $\begin{array}{l}\text { Univariate analysis. Matched by } \\
\text { age; sex; community; year of } \\
\text { serum storage; fasting status. }\end{array}$ \\
\hline $\begin{array}{l}\text { De Goede et } \\
\text { al. } 2013 \text { [38] }\end{array}$ & $\begin{array}{l}\text { The MORGEN Study } \\
\text { cohort; Nested-case } \\
\text { control; The } \\
\text { Netherlands }\end{array}$ & $\mathrm{M}+\mathrm{W}$ & 93 cases \& 93 controls & $20-65$ & ALA in CE & $\begin{array}{l}\text { Cases: } 0.53 \% \\
\text { Controls: } 0.52 \%\end{array}$ & 10.5 & $\begin{array}{l}\text { OR: } 1.02(0.71 ; 1.46) \text { for } \\
1 \text { SD increase in ALA }\end{array}$ & $\begin{array}{c}\text { Age; gender; enrollment date; } \\
\text { smoking; BMI; alcohol; high } \\
\text { educational level; diabetes; } \\
\text { hypertension; } \\
\text { hypercholesterolemia. }\end{array}$ \\
\hline $\begin{array}{l}\text { Yamagishi et } \\
\text { al. 2013 [39] }\end{array}$ & $\begin{array}{l}\text { The Minneapolis field } \\
\text { center of the ARIC } \\
\text { cohort; Follow-up; } \\
\text { United States }\end{array}$ & $\mathrm{M}+\mathrm{W}$ & $\begin{array}{l}3870 \text { subjects } \\
\text { including } 168 \text { cases }\end{array}$ & $45-65$ & $\begin{array}{l}\text { ALA in PL } \\
\text { and CE, } \\
\text { separately }\end{array}$ & $\begin{array}{l}\text { Mean: } \\
\text { CE: } 0.4 \% \\
\text { PL: } 0.1 \%\end{array}$ & 19.9 & $\begin{array}{l}\text { HR for Q4 vs. Q1: } \\
\text { CE: } 1.14(0.76 ; 1.72) \\
\text { PL: } 1.29(0.82 ; 2.02)\end{array}$ & $\begin{array}{l}\text { Age and sex. The authors stated } \\
\text { that the point estimated did not } \\
\text { materially change after } \\
\text { additional adjustment for } \\
\text { smoking status; cigarette-years } \\
\text { and alcohol intake. }\end{array}$ \\
\hline $\begin{array}{l}\text { Yaemsiri et al. } \\
2013 \text { [32] }\end{array}$ & $\begin{array}{l}\text { The WHI-OS cohort; } \\
\text { Nested case-control; } \\
\text { United status }\end{array}$ & $\mathrm{W}$ & $\begin{array}{l}964 \text { cases \& } 964 \\
\text { controls }\end{array}$ & $50-79$ & ALA in serum & $\begin{array}{l}\text { Cases: } 0.53 \% \\
\text { Controls: } 0.55 \%\end{array}$ & Not reported & $\begin{array}{l}\text { OR: } 0.90(0.74 ; 1.08) \text { for } \\
1 \text { SD increase in ALA }\end{array}$ & $\begin{array}{l}\text { Age; race; time of follow-up; } \\
\text { BMI; smoking status; diabetes; } \\
\text { aspirin use. }\end{array}$ \\
\hline $\begin{array}{l}\text { Fretts et al. } \\
2014[26]\end{array}$ & $\begin{array}{c}\text { The CHS cohort; } \\
\text { Follow-up; United } \\
\text { States }\end{array}$ & $\mathrm{M}+\mathrm{W}$ & $\begin{array}{c}2709 \text { subjects } \\
\text { including } 337 \text { cases }\end{array}$ & $\geq 65$ & ALA in PL & $\begin{array}{l}\text { Q1: } 0.09 \% \\
\text { Q3: } 0.14 \% \\
\text { Q5: } 0.22 \%\end{array}$ & $11.3^{1}$ & $\begin{array}{l}\text { Q2: } 0.92(0.65 ; 1.30) \\
\text { Q3: } 1.01(0.72 ; 1.43) \\
\text { Q4: } 0.84(0.59 ; 1.20) \\
\text { Q5: } 0.97(0.69 ; 1.36)\end{array}$ & $\begin{array}{l}\text { Age; sex; race; enrollment site; } \\
\text { education; smoking status; } \\
\text { diabetes; BMI; waist } \\
\text { circumference; physical activity; } \\
\text { alcohol; treated hypertension. }\end{array}$ \\
\hline $\begin{array}{l}\text { Daneshmand } \\
\text { et al. } 2016 \text { [40] }\end{array}$ & $\begin{array}{l}\text { The KIHD cohort; } \\
\text { Follow-up; Finland }\end{array}$ & $\mathrm{M}$ & $\begin{array}{c}1828 \text { subjects } \\
\text { including } 153 \text { cases }\end{array}$ & $42-60$ & ALA in serum & $\begin{array}{l}\text { Q1: }<0.58 \% \\
\text { Q4: }>0.87 \%\end{array}$ & 21.2 & $\begin{array}{l}\text { Q2: } 0.89(0.58 ; 1.36) \\
\text { Q3: } 0.63(0.39 ; 1.02) \\
\text { Q4: } 0.90(0.58 ; 1.41)\end{array}$ & $\begin{array}{c}\text { Age; examination year; BMI; } \\
\text { smoking status; physical activity; } \\
\text { alcohol. }\end{array}$ \\
\hline $\begin{array}{l}\text { Bork et al. } \\
2018[33]\end{array}$ & $\begin{array}{l}\text { The DCH cohort; } \\
\text { Case-cohort; } \\
\text { Denmark }\end{array}$ & $\mathrm{M}+\mathrm{W}$ & $\begin{array}{c}54,648 \text { subjects } \\
\text { including } 1735 \text { cases }\end{array}$ & $50-65$ & $\begin{array}{l}\text { ALA in } \\
\text { adipose tissue }\end{array}$ & $\begin{array}{l}\text { Q1: } 0.64 \% \\
\text { Q3: } 0.84 \% \\
\text { Q5: } 1.05 \%\end{array}$ & 13.4 & $\begin{array}{l}\text { Q2: } 0.95(0.78 ; 1.16) \\
\text { Q3: } 0.86(0.70 ; 1.06) \\
\text { Q4: } 0.93(0.76 ; 1.14) \\
\text { Q5: } 1.01(0.82 ; 1.23)\end{array}$ & $\begin{array}{l}\text { Age; sex; duration of schooling; } \\
\text { smoking; physical activity; waist } \\
\text { circumference adjusted for BMI; } \\
\text { alcohol. }\end{array}$ \\
\hline
\end{tabular}

Abbreviations: M, men; W, Women; OR, odds ratio; CI, Confidence interval; NS, not statistically significant; SD, standard deviation; MORGEN, The Monitoring Project on Risk Factors for Chronic Diseases; CE, Cholesterol ester; BMI, Body mass index; ARIC, Atherosclerosis Risk in Communities; PL, Plasma phospholipids; HR, Hazard ratio; Q, Quintile; CHS, Cardiovascular Health Study; KIHD, The Kuopio, Ischaemic Heart Disease Risk Factor Study; WHI-OS, The Women's Health Initiative Observational Follow-up Study; DCH, Diet, Cancer and Health. The presented measures of association was selected for models that included adjustment for lifestyle factors without including dietary covariates whenever possible. The lowest group of exposure was used as reference unless otherwise indicated. ${ }^{1}$ Follow-up time was calculated as person-years of follow-up divided by the total number of participants; ${ }^{2} 99 \%$ confidence interval. 
Limited data are available on biomarkers of ALA and ischemic stroke subtypes (Table 2). However, data from the DCH cohort suggested a statistically significant U-shaped association between ALA content in adipose tissue and the rate of ischemic stroke due to large artery atherosclerosis with the lowest rate observed around the median content of ALA in adipose tissue [33]. In contrast, a positive statistically non-significant association was observed between ALA content in adipose tissue and the rate of ischemic stroke due to cardioembolism, whereas no appreciable association was found between ALA in adipose tissue and ischemic stroke due to small-vessel occlusion [33].

In summary, previous studies evaluating either intake of ALA or content of ALA in blood components or adipose tissue have shown conflicting results, but most studies does not support that ALA exposure is inversely associated with ischemic stroke risk.

\section{Marine LC n-3 PUFAs and Ischemic Stroke}

Several studies have investigated the association between intake of LC n-3 PUFAs and total ischemic stroke [27,28,30,35,41-46] (Table 4) and has recently been reviewed [47]. In brief, findings from the Nurses' Health Study (NHS) indicated a statistically non-significant inverse pattern of associations between EPA+DHA intake and the rate of total ischemic stroke [30]. Analyses of ischemic stroke subtypes showed an inverse pattern of associations between EPA+DHA intake and lacunar infarctions, while no clear associations were observed for large artery occlusive infarctions [30] (Table 2). Lower rates of total ischemic stroke across quintiles of EPA+DHA intake were observed in the Swedish Mammography cohort [28] and in the Health Professionals Follow Study (HPFS) although results were not statistically significant in the latter when comparing the highest quintile of intake with the lowest [41]. In the MORGEN study, gender-specific analyses indicated lower rates of total ischemic stroke in subjects with a high EPA+DHA intake, but the confidence intervals were wide and the point estimates were not statistically significant [42]. A recent study from our groups by Venø et al. [35] investigated associations between dietary intake of total marine LC n-3 PUFAs and of individual marine n-3 PUFAs and the rate of total ischemic stroke and ischemic stroke subtypes using data from the DCH cohort. In dietary analyses, no appreciable associations were observed between intake of total and individual n-3 PUFAs, EPA or DHA and the rate of total ischemic stroke [35]. Also, in five other follow-up studies, no consistent pattern of associations between EPA+DHA intake and rate of total ischemic strokes were reported [27,43-46]. However, in the study by Venø et al. a high intake of total n-3 PUFAs, EPA and DHA was statistically significantly associated with lower rates of ischemic stroke due to large artery atherosclerosis when comparing the highest quartile of intake with the lowest [35] (Table 2). In contrast, indications of higher rates of ischemic stroke due to cardioembolism were observed with intake of LC n-3 PUFAs, whereas no consistent pattern of association was observed between LC n-3 PUFAs and the rate of ischemic stroke due small-vessel occlusions [35] (Table 2). 
Table 4. Characteristics of observational follow-up studies investigating intake of LC n-3 PUFAs and total ischemic stroke.

\begin{tabular}{|c|c|c|c|c|c|c|c|c|c|c|}
\hline $\begin{array}{l}\text { Author and } \\
\text { Study }\end{array}$ & $\begin{array}{l}\text { Study Population } \\
\text { Country }\end{array}$ & Sex & $\begin{array}{c}\text { No. of } \\
\text { Participants }\end{array}$ & $\begin{array}{l}\text { Baseline } \\
\text { Age (yrs) }\end{array}$ & $\begin{array}{c}\text { Exposure } \\
\text { Assessment }\end{array}$ & Intake (mg/d) & $\begin{array}{l}\text { No. of } \\
\text { Cases }\end{array}$ & $\begin{array}{c}\text { Follow-up } \\
\text { (yrs) }\end{array}$ & $\begin{array}{l}\text { Hazard Ratios } \\
\text { (95\% CI) }\end{array}$ & Variables Controlled for \\
\hline $\begin{array}{l}\text { Iso et al. } \\
2001[30]\end{array}$ & $\begin{array}{l}\text { The NHS cohort; } \\
\text { United States }\end{array}$ & $\mathrm{W}$ & 79,839 & $34-59$ & FFQ & $\begin{array}{l}\text { EPA+DHA } \\
\text { Q1: } 77 \\
\text { Q3: } 171 \\
\text { Q5: } 481\end{array}$ & 303 & 14 & $\begin{array}{l}\text { Q2: } 0.83(0.59 ; 1.18) \\
\text { Q3: } 0.67(0.47 ; 0.98) \\
\text { Q4: } 0.82(0.57 ; 1.18) \\
\text { Q5: } 0.71(0.46 ; 1.10)\end{array}$ & $\begin{array}{l}\text { Age; smoking status; time interval; ;oules; } \\
\text { BMI; alcohol; menopausal status; } \\
\text { post-menopausal hormone use; vigorous } \\
\text { exercise; aspirin; multivitamins; } \\
\text { hypertension; fruit; vegetables; SFA; trans } \\
\text { unsaturated fat; linoleic acid; animal } \\
\text { protein; calcium. }\end{array}$ \\
\hline $\begin{array}{l}\text { He et al. } \\
2002[41]\end{array}$ & $\begin{array}{l}\text { The HPFS cohort; } \\
\text { United States }\end{array}$ & $\mathrm{M}$ & 43,671 & $40-75$ & FFQ & $\begin{array}{l}\text { EPA+DHA } \\
\text { Q1: }<50 \\
\text { Q3: } 200-400 \\
\text { Q5: } \geq 600\end{array}$ & 377 & 12 & $\begin{array}{l}\text { Q2: } 0.56(0.35 ; 0.88) \\
\text { Q3: } 0.63(0.40 ; 0.98) \\
\text { Q4: } 0.54(0.32 ; 0.91) \\
\text { Q5: } 0.73(0.43 ; 1.25)\end{array}$ & $\begin{array}{l}\text { Age; smoking status; BMI; physical } \\
\text { activity; hypertension; aspirin; fish oil; } \\
\text { multivitamins; total calories; total fat; SFA; } \\
\text { trans-unsaturated fat; alcohol; potassium; } \\
\text { magnesium; fruit; vegetables; } \\
\text { hypercholesterolemia. }\end{array}$ \\
\hline $\begin{array}{l}\text { Yamagishi et } \\
\text { al. } 2008 \text { [46] }\end{array}$ & $\begin{array}{l}\text { The JACC cohort, } \\
\text { Japan }\end{array}$ & $\mathrm{M}+\mathrm{W}$ & 57,972 & $40-79$ & FFQ & $\begin{array}{l}\text { Total LCn-3 } \\
\text { Q1: }<1180 \\
\text { Q5: } \geq 2110\end{array}$ & 319 & 12.7 & $\begin{array}{l}\text { Q2: } 1.06(0.69 ; 1.63) \\
\text { Q3: } 1.31(0.85 ; 2.01) \\
\text { Q4: } 1.07(0.68 ; 1.69) \\
\text { Q5: } 1.17(0.71 ; 1.92)\end{array}$ & $\begin{array}{l}\text { Age; sex; hypertension; diabetes; smoking } \\
\text { status; alcohol; BMI; mental stress; } \\
\text { walking; sports; education levels; total } \\
\text { energy; intake of cholesterol, SFA, n-6 } \\
\text { PUFAs, vegetables; fruit. }\end{array}$ \\
\hline $\begin{array}{l}\text { Wallström et } \\
\text { al. } 2011 \text { [45] }\end{array}$ & $\begin{array}{l}\text { The Malmö Diet } \\
\text { and Cancer cohort, } \\
\text { Sweden }\end{array}$ & $M \& W$ & $\begin{array}{l}\text { M: } 8083 \\
\text { W: } 12,402\end{array}$ & $44-73$ & $\begin{array}{l}\text { Combined } \\
\text { FFQ, 7-day } \\
\text { diet history } \\
\text { and 1 hour } \\
\text { diet } \\
\text { interview }\end{array}$ & $\begin{array}{c}\text { EPA+DHA } \\
\text { M: } \\
\text { Q1: } 0.08 \%{ }^{1} \\
\text { Q3: } 0.19 \%^{1} \\
\text { Q5: } 0.53 \%^{1} \\
\text { W: } \\
\text { Q1: } 0.07 \%{ }^{1} \\
\text { Q3: } 0.18 \%^{1} \\
\text { Q5: } 0.49 \%^{1}\end{array}$ & $\begin{array}{l}\text { M: } 397 \\
\text { W: } 346\end{array}$ & 13.5 & $\begin{array}{c}\text { M: } \\
\text { Q2: } 1.36(0.97 ; 1.89) \\
\text { Q3: } 1.29(0.92 ; 1.80) \\
\text { Q4: } 1.12(0.80 ; 1.57) \\
\text { Q5: } 1.12(0.80 ; 1.57) \\
\text { W: } \\
\text { Q2: } 0.87(0.60 ; 1.26) \\
\text { Q3: } 0.98(0.68 ; 1.40) \\
\text { Q4: } 0.98(0.69 ; .41) \\
\text { Q5: } 1.04(0.73 ; 1.48)\end{array}$ & $\begin{array}{l}\text { Age; diet assessment method version; total } \\
\text { energy intake; season; BMI; smoking; } \\
\text { education; alcohol; systolic blood pressure, } \\
\text { anti-hypertensive treatment; } \\
\text { anti-hyperlipidemic treatment; leisure } \\
\text { time physical activity; dietary fiber. }\end{array}$ \\
\hline $\begin{array}{l}\text { Montonen et } \\
\text { al. } 2009 \text { [43] }\end{array}$ & $\begin{array}{l}\text { The Finnish Mobile } \\
\text { Clinic Health } \\
\text { Examination Survey } \\
\text { cohort, Finland }\end{array}$ & $\mathrm{M}+\mathrm{W}$ & 3958 & $40-79$ & $\begin{array}{c}\text { Dietary } \\
\text { history } \\
\text { interview }\end{array}$ & $\begin{array}{c}\text { EPA+DHA } \\
\text { Q1: } 102 \\
\text { Q4: } 655\end{array}$ & 364 & 28 & $\begin{array}{l}\text { Q2: } 0.95(0.71 ; 1.28 \\
\text { Q3: } 0.99(0.74 ; 1.33) \\
\text { Q4: } 0.91(0.66 ; 1.26)\end{array}$ & $\begin{array}{l}\text { Age; sex; energy intake; smoking; BMI; } \\
\text { physical activity; geographic area; } \\
\text { occupation; diabetes; post-hormonal } \\
\text { hormone use; hypertension; S-cholesterol; } \\
\text { intake of butter, vegetables, fruit and } \\
\text { berries. }\end{array}$ \\
\hline $\begin{array}{l}\text { Larsson et al. } \\
2012[28]\end{array}$ & $\begin{array}{l}\text { The Swedish } \\
\text { Mammography } \\
\text { cohort; Sweden }\end{array}$ & $\mathrm{W}$ & 34,670 & $49-83$ & FFQ & $\begin{array}{l}\text { EPA+DHA } \\
\text { Q1: } 131 \\
\text { Q3: } 289 \\
\text { Q5: } 559\end{array}$ & 1310 & 10.4 & $\begin{array}{l}\text { Q2: } 0.88(0.74 ; 1.04) \\
\text { Q3: } 0.84(0.70 ; 1.01) \\
\text { Q4: } 0.83(0.69 ; 0.99) \\
\text { Q5: } 0.83(0.69 ; 0.99)\end{array}$ & $\begin{array}{l}\text { Age; smoking status and pack-years of } \\
\text { smoking; education; BMI; physical } \\
\text { activity; hypertension; diabetes; aspirin; } \\
\text { family history of MI; intakes of alcohol, } \\
\text { protein, fiber and cholesterol. }\end{array}$ \\
\hline
\end{tabular}


Table 4. Cont.

\begin{tabular}{|c|c|c|c|c|c|c|c|c|c|c|}
\hline $\begin{array}{l}\text { Author and } \\
\text { Study }\end{array}$ & $\begin{array}{l}\text { Study Population } \\
\text { Country }\end{array}$ & Sex & $\begin{array}{c}\text { No. of } \\
\text { Participants }\end{array}$ & $\begin{array}{l}\text { Baseline } \\
\text { Age (yrs) }\end{array}$ & $\begin{array}{c}\text { Exposure } \\
\text { Assessment }\end{array}$ & Intake (mg/d) & $\begin{array}{l}\text { No. of } \\
\text { Cases }\end{array}$ & $\begin{array}{c}\text { Follow-up } \\
\text { (yrs) }\end{array}$ & $\begin{array}{l}\text { Hazard Ratios } \\
(95 \% \text { CI })\end{array}$ & Variables Controlled for \\
\hline $\begin{array}{l}\text { De Goede et } \\
\text { al. 2012 [42] }\end{array}$ & $\begin{array}{l}\text { The MORGEN } \\
\text { Study cohort; The } \\
\text { Netherlands }\end{array}$ & $\mathrm{M} \& \mathrm{~W}$ & $\begin{array}{c}\text { M: 8,988W: } \\
\text { 11,081 }\end{array}$ & $20-65$ & FFQ & $\begin{array}{c}\text { EPA+DHA } \\
\text { M: } \\
\text { Q1: } 44 \\
\text { Q4: } 241 \\
\text { W: } \\
\text { Q1: } 36 \\
\text { Q4: } 225\end{array}$ & $\begin{array}{l}\text { M: } 80 \\
\text { W: } 64\end{array}$ & 10.5 & $\begin{array}{c}\text { M: } \\
\text { Q2: } 0.93(0.50 ; 1.74) \\
\text { Q3: } 0.87(0.46 ; 1.65) \\
\text { Q4: } 0.85(0.45 ; 1.60) \\
\text { W: } \\
\text { Q2: } 0.98(0.50 ; 1.91) \\
\text { Q3: } 0.98(0.50 ; 1.93) \\
\text { Q4: } 0.62(0.29 ; 1.35)\end{array}$ & $\begin{array}{l}\text { Age; smoking; BMI; educational level; } \\
\text { parental history of MI; alcohol; total } \\
\text { energy intake; intake of fiber, vitamin C, } \\
\text { beta-carotene, SFAs, trans fatty acids, } \\
\text { MUFAs, linoleic acid and ALA. }\end{array}$ \\
\hline $\begin{array}{l}\text { Kippler et al. } \\
\text { 2016 [44] }\end{array}$ & $\begin{array}{l}\text { The Cohort of } \\
\text { Swedish Men, } \\
\text { Sweden }\end{array}$ & M & 39,948 & $45-79$ & FFQ & $\begin{array}{l}\text { EPA+DHA } \\
\text { Q1:180 } \\
\text { Q4: } 730\end{array}$ & 2286 & 12 & $\begin{array}{l}\text { Q2: } 1.02(0.90 ; 1.16) \\
\text { Q3: } 0.98(0.85 ; 1.13) \\
\text { Q4: } 1.09(0.94 ; 1.26)\end{array}$ & $\begin{array}{l}\text { Age; educational level; family history of } \\
\text { MI; high cholesterol; hypertension; atrial } \\
\text { fibrillation; aspirin; BMI; smoking status; } \\
\text { alcohol; physical activity; fish oil } \\
\text { supplements, energy intake, intake of fruit, } \\
\text { vegetables, red and processed meat, dairy } \\
\text { products, SFAs, MeHg and PCB. }\end{array}$ \\
\hline $\begin{array}{l}\text { Rhee et al. } \\
2017[27]\end{array}$ & $\begin{array}{l}\text { The WHS cohort; } \\
\text { United States }\end{array}$ & W & 38,392 & $\geq 45$ & FFQ & $\begin{array}{l}\text { EPA+DHA } \\
\text { Q1: } 0.06 \%{ }^{1} \\
\text { Q3: } 0.16 \%^{1} \\
\text { Q5: } 0.40 \%^{1}\end{array}$ & 807 & $18.2^{2}$ & $\begin{array}{l}\text { Q2: } 0.99(0.80 ; 1.23) \\
\text { Q3: } 0.90(0.72 ; 1.14) \\
\text { Q4: } 0.92(0.74 ; 1.14) \\
\text { Q5: } 1.04(0.84 ; 1.29)\end{array}$ & $\begin{array}{l}\text { Age; randomized treatment assignment; } \\
\text { BMI; smoking; alcohol; physical activity; } \\
\text { oral conceptive use; HRT; multivitamin } \\
\text { use; energy intake; family history of MI; } \\
\text { hypertension; high cholesterol; diabetes. }\end{array}$ \\
\hline $\begin{array}{l}\text { Venø et al. } \\
2018[35]\end{array}$ & $\begin{array}{l}\text { The DCH cohort; } \\
\text { Denmark }\end{array}$ & $\mathrm{M}+\mathrm{W}$ & 55,338 & $50-65$ & FFQ & $\begin{array}{l}\text { Total LCn-3 } \\
\text { median: } \sim 700\end{array}$ & 1879 & 13.5 & $\begin{array}{l}\text { Q2: } 1.06(0.93 ; 1.21) \\
\text { Q3: } 1.06(0.93 ; 1.21) \\
\text { Q4: } 1.06(0.93 ; 1.20)\end{array}$ & $\begin{array}{l}\text { Age; sex; education; waist circumference } \\
\text { adjusted by BMI; smoking; physical } \\
\text { activity; alcohol; alcohol abstain. }\end{array}$ \\
\hline
\end{tabular}

Abbreviations: NHS, Nurses's Health Study; W, Women; FFQ, Food Frequency Questionnaire; EPA, Eicosapentaenoic acid; DHA, Docosahexaenoic acid; Q, Quintile; CI, Confidence interval; BMI, Body mass index; SFA, Saturated fatty acids; M, men; HPFS, Health Professionals Follow-up Study; JACC, Japan Collaborative Cohort Study for Evaluation of Cancer Risk Study; LCn-3, Long-chain n-3 PUFAs; MI, Myocardial infarction; MORGEN, Monitoring Project on Risk Factors for Chronic Diseases; MUFA, Monounsaturated fatty acids; MeHg, Methylmercery; PCB, Polychlorinated biphenyls; WHS, Women's Health Study; DCH, Diet, Cancer and Health. The presented measures of association was selected for models that Methylmercery; PCB, Polychlorinated biphenyls; WHS, Women's Health Study; DCH, Diet, Cancer and Health. The presented measures of association was selected for models that
included adjustment for lifestyle factors without including dietary covariates whenever possible. The lowest group of exposure was used as reference unless otherwise indicated. 1 Percentage of total energy intake; ${ }^{2}$ Follow-up time was calculated as person years divided by the total number of participants. 
Some biomarker studies have investigated the associations between levels of LC $\mathrm{n}-3$ PUFAs in blood compartments or adipose tissue and the risk of ischemic stroke [31,32,34,35,38-40,48] (Table 5). Thus, a the content of EPA, DPA and DHA in plasma phospholipids was analysed in relation to ischemic stroke and its subtypes using pooled data from the CHS, NHS and HPFS [34]. Lower rates of total ischemic stroke was observed when comparing the highest quintile of content of DPA and DHA with the lowest, respectively, while no appreciable association was found for EPA [34]. However, the associations seemed to depend on the ischemic stroke subtype in question (Table 2). In analyses of ischemic stroke subtypes, DHA was associated with the rate of atherothrombotic strokes (large artery and small-vessel infarctions), whereas no association was found for EPA and DPA. However, DPA was associated with lower rates of cardioembolic strokes, whereas no associations were observed between EPA or DHA and the rate of cardioembolic stroke [34]. Few other studies have supported that circulating biomarkers of LC n-3 PUFAs might be associated with a lower risk of ischemic stroke although results have not been consistent $[31,32,38,40,48]$ (Table 5). 
Table 5. Characteristics of observational follow-up and nested case-control studies investigating biomarkers of LC n-3 PUFAs and total ischemic stroke.

\begin{tabular}{|c|c|c|c|c|c|c|c|c|c|}
\hline $\begin{array}{l}\text { Author and } \\
\text { Study }\end{array}$ & $\begin{array}{l}\text { Study Population, } \\
\text { Design and Country }\end{array}$ & Sex & $\begin{array}{l}\text { No. of Partici-pants } \\
\text { and Cases }\end{array}$ & $\begin{array}{l}\text { Baseline Age } \\
\text { (yrs) }\end{array}$ & Biomarker & $\begin{array}{c}\text { Concentrations } \\
(\%)\end{array}$ & $\begin{array}{c}\text { Follow-up } \\
\text { (yrs) }\end{array}$ & $\begin{array}{c}\text { Measure of } \\
\text { Association }(95 \% \mathrm{CI})\end{array}$ & Variables Controlled for \\
\hline $\begin{array}{l}\text { Iso et al. } 2002 \\
{[31]}\end{array}$ & $\begin{array}{c}\text { A Japanese } \\
\text { cardio-vascular risk } \\
\text { survey population; } \\
\text { Nested case-control; } \\
\text { Japan }\end{array}$ & $\mathrm{M}+\mathrm{W}$ & $\begin{array}{l}122 \text { cases \& } 366 \\
\text { controls }\end{array}$ & $40-85$ & $\begin{array}{l}\text { EPA, DPA and } \\
\text { DHA in serum } \\
\text { (separately) }\end{array}$ & $\begin{array}{l}\text { EPA: } \\
\text { Cases: } 3.6 \\
\text { Controls: } 3.7 \\
\text { DHA: } \\
\text { Cases: } 4.5 \\
\text { Controls: } 4.4 \\
\end{array}$ & Not reported & $\begin{array}{l}\text { OR: } 1.01 \text { (NS, CI not } \\
\text { reported) per } 1 \text { SD } \\
\text { increase in EPA OR: } \\
1.03 \text { (NS, CI not } \\
\text { reported) per 1 SD } \\
\text { increase in DHA }\end{array}$ & $\begin{array}{l}\text { Univariate analysis. Matched by age; } \\
\text { sex; community; year of serum storage; } \\
\text { fasting status. }\end{array}$ \\
\hline $\begin{array}{l}\text { De Goede et } \\
\text { al. } 2013[38]\end{array}$ & $\begin{array}{l}\text { The MORGEN Study } \\
\text { cohort; Nested-case } \\
\text { control; The } \\
\text { Netherlands }\end{array}$ & $\mathrm{M}+\mathrm{W}$ & 93 cases \& 93 controls & $20-65$ & $\begin{array}{c}\mathrm{EPA}+\mathrm{DHA} \text { in } \\
\mathrm{CE}\end{array}$ & $\begin{array}{l}\text { Cases: } 1.57 \\
\text { Controls: } 1.25\end{array}$ & 10.5 & $\begin{array}{l}\text { OR: } 1.33(0.96 ; 1.84) \text { for } \\
1 \text { SD increase in } \\
\text { EPA+DHA }\end{array}$ & $\begin{array}{l}\text { Age; gender; enrollment date; smoking; } \\
\text { BMI; alcohol; high educational level; } \\
\text { diabetes; hypertension; } \\
\text { hypercholesterolemia. }\end{array}$ \\
\hline $\begin{array}{l}\text { Mozaffarian et } \\
\text { al. } 2013[48]\end{array}$ & $\begin{array}{c}\text { The CHS cohort; } \\
\text { Follow-up; United } \\
\text { States }\end{array}$ & $\mathrm{M}+\mathrm{W}$ & $\begin{array}{l}2692 \text { subjects } \\
\text { including } 319 \text { cases }\end{array}$ & $\geq 65$ & $\begin{array}{l}\text { Total LCn-3, } \\
\text { EPA, DPA and } \\
\text { DHA in PL }\end{array}$ & $\begin{array}{l}\text { Total LCn-3 } \\
\text { Q1: } 3.17 \\
\text { Q3: } 4.21 \\
\text { Q5: } 6.04\end{array}$ & $11.5^{1}$ & $\begin{array}{l}\text { Q2: } 0.88(0.63 ; 1.23) \\
\text { Q3: } 0.77(0.54 ; 1.08) \\
\text { Q4: } 0.93(0.66 ; 1.31) \\
\text { Q5: } 0.63(0.43 ; 0.94)\end{array}$ & $\begin{array}{l}\text { Age; sex; race; enrollment site; fatty acid } \\
\text { measurement batch; education; smoking } \\
\text { status; diabetes; atrial fibrillation; } \\
\text { treated hypertension; leisure time } \\
\text { physical activity; BMI; waist } \\
\text { circumference; alcohol; intake of broiled } \\
\text { and baked fish, fried fish, red meat, } \\
\text { fruits, vegetables and fiber. }\end{array}$ \\
\hline $\begin{array}{l}\text { Yaemsiri et al. } \\
2013 \text { [32] }\end{array}$ & $\begin{array}{l}\text { The WHI-OS cohort; } \\
\text { Nested case-control; } \\
\text { United status }\end{array}$ & W & $\begin{array}{l}964 \text { cases \& } 964 \\
\text { controls }\end{array}$ & $50-79$ & $\begin{array}{l}\text { EPA, DPA and } \\
\text { DHA in serum } \\
\text { (separately) }\end{array}$ & $\begin{array}{c}\text { EPA: } \\
\text { Cases: } 0.56 \\
\text { Controls: } 0.60 \\
\text { DHA: } \\
\text { Cases: } 1.75 \\
\text { Controls: } 1.97 \\
\end{array}$ & Not reported & $\begin{array}{l}\text { OR: } 0.84(0.70 ; 1.01) \text { for } \\
1 \text { SD increase in EPA }{ }^{2} \\
\text { OR: } 0.72(0.59 ; 0.87) \text { for } \\
1 \text { SD increase in DHA }{ }^{2}\end{array}$ & $\begin{array}{l}\text { Age; race; time of follow-up; BMI; } \\
\text { smoking status; diabetes; aspirin. }\end{array}$ \\
\hline $\begin{array}{l}\text { Yamagishi et } \\
\text { al. 2013 [39] }\end{array}$ & $\begin{array}{l}\text { The Minneapolis field } \\
\text { center of the ARIC } \\
\text { cohort; Follow-up; } \\
\text { United States }\end{array}$ & $\mathrm{M}+\mathrm{W}$ & $\begin{array}{l}3870 \text { subjects } \\
\text { including } 168 \text { cases }\end{array}$ & $45-65$ & $\begin{array}{l}\text { Total LCn-3, } \\
\text { EPA and DHA } \\
\text { in PL and CE } \\
\text { (separately) }\end{array}$ & $\begin{array}{c}\text { Total LCn-3 } \\
\text { CE: } \\
\text { Q1: } 0.22-0.77 \\
\text { Q4: } 1.15-6.02 \\
\text { PL: } \\
\text { Q1: } 1.51-3.57 \\
\text { Q4: } 4.75-13.5\end{array}$ & 19.9 & $\begin{array}{l}\text { HR for Q4 vs. Q1: } \\
\text { CE: } 1.16(0.75 ; 1.79) \\
\text { PL: } 0.86(0.56 ; 1.32)\end{array}$ & $\begin{array}{l}\text { Age and sex. The authors stated that the } \\
\text { point estimated did not materially } \\
\text { change after additional adjustment for } \\
\text { smoking status; cigarette-years and } \\
\text { alcohol intake. }\end{array}$ \\
\hline
\end{tabular}


Table 5. Cont.

\begin{tabular}{|c|c|c|c|c|c|c|c|c|c|}
\hline $\begin{array}{l}\text { Author and } \\
\text { Study }\end{array}$ & $\begin{array}{c}\text { Study Population, } \\
\text { Design and Country }\end{array}$ & Sex & $\begin{array}{l}\text { No. of Partici-pants } \\
\text { and Cases }\end{array}$ & $\begin{array}{l}\text { Baseline Age } \\
\text { (yrs) }\end{array}$ & Biomarker & $\begin{array}{c}\text { Concentrations } \\
(\%)\end{array}$ & $\begin{array}{c}\text { Follow-up } \\
\text { (yrs) }\end{array}$ & $\begin{array}{c}\text { Measure of } \\
\text { Association }(95 \% \mathrm{CI})\end{array}$ & Variables Controlled for \\
\hline $\begin{array}{l}\text { Daneshmand } \\
\text { et al. } 2016[40]\end{array}$ & $\begin{array}{l}\text { The KIHD cohort; } \\
\text { Follow-up; Finland }\end{array}$ & M & $\begin{array}{c}1828 \text { subjects } \\
\text { including } 153 \text { cases }\end{array}$ & $42-60$ & $\begin{array}{l}\text { Total LCn-3, } \\
\text { EPA, DPA and } \\
\text { DHA in serum }\end{array}$ & $\begin{array}{l}\text { Total LCn-3 } \\
\text { Q1: }<3.63 \% \\
\text { Q4: }>5.34 \%\end{array}$ & 21.2 & $\begin{array}{l}\text { Q2: } 0.66(0.41 ; 1.07) \\
\text { Q3: } 0.91(0.58 ; 1.42) \\
\text { Q4: } 0.98(0.64 ; 1.51)\end{array}$ & $\begin{array}{l}\text { Age; examination year; BMI; smoking } \\
\text { status; physical activity; alcohol. }\end{array}$ \\
\hline $\begin{array}{l}\text { Saber et al. } \\
2017 \text { [34] }\end{array}$ & $\begin{array}{l}\text { Pooled analysis of the } \\
\text { NHS and HFPS } \\
\text { (nested case-control), } \\
\text { and the CHS } \\
\text { (follow-up) cohorts, } \\
\text { United States }\end{array}$ & $\mathrm{M}+\mathrm{W}$ & $\begin{array}{l}\text { A total of } 953 \text { cases } \\
\text { and } 437 \text { controls from } \\
\text { the NHS and HFPS } \\
\text { and } 3941 \text { subjects } \\
\text { from the CHS }\end{array}$ & $\begin{array}{l}\text { NHS: } \\
30-55 \\
\text { HFPS: } \\
\text { 40-75 } \\
\text { CHS: } \\
\geq 65\end{array}$ & $\begin{array}{l}\text { EPA, DPA and } \\
\text { DHA in PL } \\
\text { (separately) }\end{array}$ & $\begin{array}{l}\text { Cohort-specific fatty } \\
\text { acid content in } \\
\text { quartiles can be } \\
\text { found in the paper. }\end{array}$ & $\begin{array}{c}\text { NHS+HPFS: } \\
11.2 \\
\text { CHS: } 8.3\end{array}$ & $\begin{array}{c}\text { EPA: } \\
\text { Q2: } 0.95(0.77 ; 1.18) \\
\text { Q3: } 0.93(0.74 ; 1.16) \\
\text { Q4: } 0.94(0.77 ; 1.19) \\
\text { DHA: } \\
\text { Q2: } 0.93(0.75 ; 1.14) \\
\text { Q3: } 0.85(0.71 ; 1.10) \\
\text { Q4: } 0.80(0.63 ; 1.00)\end{array}$ & $\begin{array}{l}\text { Age; race; sex; smoking status; physical } \\
\text { activity; alcohol; hypertension; family } \\
\text { history of diabetes; parental history of } \\
\text { CVD; menopausal status; } \\
\text { postmenopausal hormone use; BMI; } \\
\text { aspirin; intake of processed and } \\
\text { unprocessed meat, fruits and vegetables. }\end{array}$ \\
\hline $\begin{array}{l}\text { Venø et al. } \\
2019 \text { [35] }\end{array}$ & $\begin{array}{l}\text { The DCH cohort; } \\
\text { Case-cohort, } \\
\text { Denmark }\end{array}$ & $\mathrm{M}+\mathrm{W}$ & $\begin{array}{l}55,338 \text { subjects } \\
\text { including } 1755 \text { cases }\end{array}$ & $50-65$ & $\begin{array}{l}\text { Total LCn-3, } \\
\text { EPA, DPA and } \\
\text { DPA in } \\
\text { adipose tissue }\end{array}$ & $\begin{array}{l}\text { Total LCn-3: } \\
\text { Q1: } 0.42 \\
\text { Q4: } 0.94\end{array}$ & 13.5 & $\begin{array}{l}\text { Q2: } 0.98(0.82 ; 1.17) \\
\text { Q3: } 1.12(0.94 ; 1.33) \\
\text { Q4: } 1.08(0.90 ; 1.30)\end{array}$ & $\begin{array}{l}\text { Age; sex; education; waist circumference } \\
\text { adjusted for BMI; smoking; physical } \\
\text { activity; alcohol and alcohol abstain. }\end{array}$ \\
\hline
\end{tabular}

Abbreviations: M, men; W, Women; EPA, Eicosapentaenoic acid; DPA, Docosapentaenoic acid; DHA, Docosahexaenoic acid; OR, Odds ratio; CI, Confidence interval; NS, Not statistically significant; SD, Standard deviation; MORGEN, The Monitoring Project on Risk Factors for Chronic Diseases; CE, Cholesterol esters; BMI, Body mass index; CHS, Cardiovascular Health Study; LC n-3, Long-chain n-3 PUFAs, PL, Plasma phospholipids; Q, Quintile; WHI-OS, Women's Health Initiative Observational Follow-up Study; KIHD, The Kuopio, Ischaemic Heart Disease Risk Factor Study; NHS, Nurses' Health Study; HPFS, Health Professionals Follow-up Study; DCH, Diet, Cancer and Health. The presented measures of association was selected for models that included adjustment for lifestyle factors without including dietary covariates whenever possible. The lowest group of exposure was used as reference unless otherwise indicated. ${ }^{1}$ Follow-up time was calculated as person years divided by the total number of participants; ${ }^{2} 99 \%$ confidence interval. 
The associations between adipose tissue content of LC n-3 PUFAs and the risk of ischemic stroke and ischemic stroke subtypes were investigated based on data from the DCH cohort and no appreciable association was observed between total adipose tissue of LC n-3 PUFAs and the rate of total ischemic stroke [35]. However, the EPA content in adipose tissue was inversely associated with the rate of total ischemic stroke when comparing the highest with the lowest quintile [35]. In contrast, adipose tissue content of DPA seemed to be associated with a higher rate of total ischemic stroke, whereas no association was found for DHA [35]. In analyses of ischemic stroke subtypes, the content of EPA in adipose tissue was inversely associated with the rate of ischemic stroke due to large artery atherosclerosis and small-vessel occlusions, while a higher rate of ischemic stroke due to cardioembolism was noted when comparing the highest quartile with the lowest although not statistically significant [35] (Table 2). Adipose tissue content of DPA and DHA was associated with higher rates of ischemic stroke caused by cardioembolism whereas no consistent pattern of association was found for ischemic stroke caused by large artery atherosclerosis or small-vessel occlusions [35] (Table 2).

In summary, several large prospective studies evaluating EPA and/or DHA intake or their content in blood components or adipose tissue have suggested an inverse association although results have not been consistent. The associations between LC n-3 PUFAs and ischemic stroke subtypes may differ with the most beneficial results observed in subjects with strokes of atherosclerotic etiology.

\section{LA and Ischemic Stroke}

Studies of the association between dietary intake of LA and the rate of ischemic stroke are sparse (Table 6). In analyses based on data from the Swedish Mammography Cohort, no association was observed between intake of LA+AA and the rate of total ischemic stroke [28]. Also, no clear association was observed between LA+AA intake and the rate of total ischemic stroke in another Swedish cohort [45]. However, the intake of LA and AA was not analysed separately and the macronutrient(s) to be replaced for n-6 PUFAs was not specified in these studies. In contrast to n-3 PUFAs, LA constitute a significant proportion of total energy intake and a higher intake of LA must necessarily be accompanied by a lower intake of other macronutrients in an isocaloric setting. In a follow-up study using statistical substitution models we therefore investigated the risk of total ischemic stroke and ischemic stroke subtypes with a $5 \%$ higher intake of LA and a concomitant lower intake from saturated fatty acids (SFAs), monounsaturated fatty acids (MUFAs) or glycemic carbohydrates using data from the DCH cohort [36]. This study suggested that replacing MUFAs or glycemic carbohydrates with LA might be associated with a lower risk of total ischemic stroke although not statistically significantly [36]. Further, in analyses of ischemic stroke subtypes, replacement of SFA with LA indicated a lower rate of ischemic stroke due to large artery atherosclerosis and replacement of glycemic carbohydrates with LA indicated a lower rate of ischemic stroke due to small-vessel occlusions, although the observed associations were not statistically significant [36] (Table 2). A statistically significant lower rate of ischemic stroke due to small-vessel occlusions was observed when LA replaced MUFAs [36] (Table 2). 
Table 6. Characteristics of observational follow-up studies investigating intake of linoleic acid (LA) and total ischemic stroke.

\begin{tabular}{|c|c|c|c|c|c|c|c|c|c|c|}
\hline $\begin{array}{c}\text { Author and } \\
\text { Study }\end{array}$ & $\begin{array}{l}\text { Study Population } \\
\text { and Country }\end{array}$ & Sex & $\begin{array}{c}\text { No. of } \\
\text { Participants }\end{array}$ & $\begin{array}{l}\text { Baseline } \\
\text { Age (yrs) }\end{array}$ & $\begin{array}{c}\text { Exposure } \\
\text { Assessment }\end{array}$ & Intake (g/d) & $\begin{array}{l}\text { No. of } \\
\text { Cases }\end{array}$ & $\begin{array}{c}\text { Follow-up } \\
\text { (yrs) }\end{array}$ & $\begin{array}{l}\text { Hazard Ratios } \\
\text { (95\% CI) }\end{array}$ & Variables Controlled for \\
\hline $\begin{array}{l}\text { Larsson et al. } \\
2012[28]\end{array}$ & $\begin{array}{l}\text { The Swedish } \\
\text { Mammography } \\
\text { Cohort, Sweden }\end{array}$ & W & 34,670 & $49-83$ & FFQ & $\begin{array}{l}\text { LA+AA } \\
\text { Q1: } 4.7 \\
\text { Q3: } 6.0 \\
\text { Q5: } 8.0\end{array}$ & 1310 & 10.4 & $\begin{array}{l}\text { Q2: } 0.98(0.84 ; 1.16) \\
\text { Q3: } 0.96(0.81 ; 1.14) \\
\text { Q4: } 1.02(0.85 ; 1.21) \\
\text { Q5: } 0.96(0.80 ; 1.15)\end{array}$ & $\begin{array}{l}\text { Age; smoking status and pack-years of } \\
\text { smoking; education; BMI; physical } \\
\text { activity; hypertension; diabetes; aspirin; } \\
\text { family history of MI; intakes of alcohol, } \\
\text { protein, fiber and cholesterol. }\end{array}$ \\
\hline $\begin{array}{l}\text { Wallstrøm et } \\
\text { al. } 2012 \text { [45] }\end{array}$ & $\begin{array}{l}\text { The Malmö Diet } \\
\text { and Cancer cohort, } \\
\text { Sweden }\end{array}$ & $\mathrm{M} \& \mathrm{~W}$ & $\begin{array}{c}\text { M: } 8083 \\
\text { W: } 12,402\end{array}$ & $44-73$ & $\begin{array}{l}\text { Combined } \\
\text { FFQ, 7-day } \\
\text { diet history } \\
\text { and } 1 \mathrm{~h} \text { diet } \\
\text { interview }\end{array}$ & $\begin{array}{c}\text { LA+AA: } \\
\text { Q1: } 3.5 \%^{1} \\
\text { Q3: } 5 . \%^{1} \\
\text { Q5: } 7.1 \%^{1} \\
\text { W: } \\
\text { Q1: } 3.3 \%^{1} \\
\text { Q3: } 4.7 \%^{1} \\
\text { Q5: } 6.7 \%^{1}\end{array}$ & $\begin{array}{l}\text { M: } 397 \mathrm{~W}: \\
\quad 346\end{array}$ & 13.5 & $\begin{array}{c}\text { M: } \\
\text { Q2: } 1.12(0.82 ; 1.52) \\
\text { Q3: } 1.02(0.741 .39) \\
\text { Q4: } 1.10(0.80 ; 1.50) \\
\text { Q5: } 1.16(0.84 ; 1.58) \\
\text { W: } \\
\text { Q2: } 0.99(0.72 ; 1.36) \\
\text { Q3: } 0.86(0.62 ; 1.19) \\
\text { Q4: } 1.02(0.73 ; 1.41) \\
\text { Q5: } 0.81(0.57 ; 1.14)\end{array}$ & $\begin{array}{l}\text { Age; diet assessment method version; total } \\
\text { energy intake; season; BMI; smoking; } \\
\text { education; alcohol; systolic blood pressure, } \\
\text { anti-hypertensive treatment; } \\
\text { anti-hyperlipidemic treatment; leisure } \\
\text { time physical activity; dietary fiber. }\end{array}$ \\
\hline $\begin{array}{l}\text { Venø et al. } \\
2017 \text { [36] }\end{array}$ & $\begin{array}{l}\text { The DCH cohort; } \\
\text { Denmark }\end{array}$ & $\mathrm{M}+\mathrm{W}$ & 55,338 & $50-65$ & FFQ & Median 10.7 & 1879 & 13.5 & $\begin{array}{c}\text { LA for SFA: } \\
0.98(0.83 ; 1.16) \\
\text { LA for MUFA } \\
0.80(0.63 ; 1.02) \\
\text { LA for glycemic } \\
\text { carbohydrates: } \\
0.92(0.78 ; 1.09)\end{array}$ & $\begin{array}{l}\text { Age; sex; total energy intake; education } \\
\text { status; physical activity; waist } \\
\text { circumference adjusted for BMI; alcohol; } \\
\text { alcohol abstain; smoking. }\end{array}$ \\
\hline
\end{tabular}

Abbreviations: M, men; W, Women; FFQ, Food Frequency Questionnaire; AA, Arachidonic acid; Q, Quintile; CI, Confidence interval; BMI, Body mass index; MI, Myocardial infarction; SFA, Saturated fatty acids; MUFA, Monounsaturated fatty acids; DCH, Diet Cancer and Health. The presented measures of association was selected for models that included adjustment for lifestyle factors without including dietary covariates whenever possible. The lowest group of exposure was used as reference unless otherwise indicated. ${ }^{1}$ Percentage of total energy intake. 
Several prospective biomarker studies have supported that circulating or adipose tissue content of LA may be associated with a lower risk of ischemic stroke although results have not been consistent $[23,31,32,37-40,49]$ (Table 7). A previous study by Iso et al. suggested a lower odds of total ischemic stroke and lacunar infarctions per $1 \mathrm{SD}$ increase in LA content in serum [31]. Also, in analyses based on data from the ARIC Study, a statistically significant inverse association between LA content in plasma cholesterol esters and the rate of total ischemic stroke and indications of an inverse association was observed between LA content in plasma phospholipids and the rate of total ischemic stroke [39]. Also, several other studies have indicated a lower risk of total ischemic stroke with the content of LA in serum, plasma phospholipids and cholesterol esters, but these findings were not statistically significant and not entirely consistent $[32,38,40,49]$. However, a large case-cohort study based on data from the DCH study found a dose-dependent inverse association between adipose tissue content of LA and the rate of total ischemic stroke [37]. In analyses of ischemic stroke subtypes, adipose tissue content of LA was inversely associated with the rate of ischemic stroke due to large artery atherosclerosis and indications of lower rates of ischemic stroke due to small-vessel occlusions was observed, whereas no clear association was found between LA in adipose and the rate of ischemic stroke due to cardioembolism [37]. Recently, a harmonized individual-level analysis based on prospective data from the Fatty Acid and Outcome Research Consortium (FORCE) found an overall lower rate of total ischemic stroke with higher levels of LA in blood compartments and adipose tissue in analyses including 3705 incident ischemic stroke cases [23]. 
Table 7. Characteristics of observational follow-up and nested case-control studies investigating biomarkers of linoleic acid (LA) and total ischemic stroke.

\begin{tabular}{|c|c|c|c|c|c|c|c|c|c|}
\hline $\begin{array}{l}\text { Author and } \\
\text { Study }\end{array}$ & $\begin{array}{c}\text { Study Population, } \\
\text { Design and Country }\end{array}$ & Sex & $\begin{array}{l}\text { No. of Partici-pants } \\
\text { and Cases }\end{array}$ & $\begin{array}{c}\text { Baseline Age } \\
\text { (yrs) }\end{array}$ & Biomarker & $\begin{array}{c}\text { Concentrations } \\
(\%)\end{array}$ & $\begin{array}{c}\begin{array}{c}\text { Follow-up } \\
\text { (yrs) }\end{array} \\
\end{array}$ & $\begin{array}{c}\text { Measure of } \\
\text { Association }(95 \% \mathrm{CI}) \\
\end{array}$ & Variables Controlled for \\
\hline $\begin{array}{l}\text { Iso et al. } 2002 \\
{[31]}\end{array}$ & $\begin{array}{c}\text { A Japanese } \\
\text { cardiovascular risk } \\
\text { survey population; } \\
\text { Nested case-control; } \\
\text { Japan }\end{array}$ & $\mathrm{M}+\mathrm{W}$ & $\begin{array}{l}122 \text { cases \& } 366 \\
\text { controls }\end{array}$ & $40-85$ & LA in serum & $\begin{array}{l}\text { Cases: } 26.1 \% \\
\text { Controls: } 27.9 \%\end{array}$ & Not reported & $\begin{array}{l}\text { OR } 0.66(0.49 ; 0.88) \text { per } \\
1 \text { SD increase in LA }\end{array}$ & $\begin{array}{l}\text { Age; sex; community; year of serum } \\
\text { storage; fasting status; BMI; cigarette } \\
\text { smoking status; alcohol; hypertension; } \\
\text { serum cholesterol levels, triglyceride } \\
\text { and glucose levels. }\end{array}$ \\
\hline $\begin{array}{l}\text { Yaemsiri et al. } \\
2013 \text { [32] }\end{array}$ & $\begin{array}{l}\text { The WHI-OS cohort; } \\
\text { Nested case-control; } \\
\text { United status }\end{array}$ & $\mathrm{W}$ & $\begin{array}{l}964 \text { cases \& } 964 \\
\text { controls }\end{array}$ & 50-79 & LA in serum & $\begin{array}{l}\text { Cases: } 26.7 \% \\
\text { Controls: } 27.1 \%\end{array}$ & Not reported & $\begin{array}{l}\text { OR: } 0.92(0.79 ; 1.08) \text { for } \\
1 \text { SD increase in LA }{ }^{2}\end{array}$ & $\begin{array}{l}\text { Age; race; time of follow-up; BMI; } \\
\text { smoking status; diabetes; aspirin. }\end{array}$ \\
\hline $\begin{array}{l}\text { Yamagishi et } \\
\text { al. 2013 [39] }\end{array}$ & $\begin{array}{l}\text { The Minneapolis field } \\
\text { center of the ARIC } \\
\text { cohort; Follow-up; } \\
\text { United States }\end{array}$ & $\mathrm{M}+\mathrm{W}$ & $\begin{array}{l}3870 \text { subjects } \\
\text { including } 168 \text { cases }\end{array}$ & $45-65$ & $\begin{array}{l}\text { LA in PL and } \\
\text { CE } \\
\text { (separately) }\end{array}$ & $\begin{array}{c}\text { CE: } \\
\text { Q1: } 21.5-51.3 \% \\
\text { Q4: } 57.4-68.2 \% \\
\text { PL: } \\
\text { Q1: } 9.0-20.3 \% \\
\text { Q4: } 23.7-32.4 \% \\
\end{array}$ & 19.9 & $\begin{array}{l}\text { HR for Q4 vs. Q1: } \\
\text { CE: } 0.64(0.43 ; 0.97) \\
\text { PL: } 0.69(0.45 ; 1.05)\end{array}$ & $\begin{array}{l}\text { Age and sex. The authors stated that } \\
\text { the point estimated did not materially } \\
\text { change after additional adjustment for } \\
\text { smoking status; cigarette-years and } \\
\text { alcohol intake. }\end{array}$ \\
\hline $\begin{array}{l}\text { De Goede et } \\
\text { al. } 2013[38]\end{array}$ & $\begin{array}{l}\text { The MORGEN Study } \\
\text { cohort; Nested-case } \\
\text { control; The } \\
\text { Netherlands }\end{array}$ & $\mathrm{M}+\mathrm{W}$ & 93 cases \& 93 controls & $20-65$ & LA in CE & $\begin{array}{l}\text { Cases: } 54.2 \% \\
\text { Controls: } 55.4 \%\end{array}$ & 10.5 & $\begin{array}{l}\text { OR: } 0.81(0.54 ; 1.24) \text { for } \\
1 \text { SD increase in LA }\end{array}$ & $\begin{array}{l}\text { Age; gender; enrollment date; } \\
\text { smoking; BMI; alcohol; high } \\
\text { educational level; diabetes; } \\
\text { hypertension; hypercholesterolemia. }\end{array}$ \\
\hline $\begin{array}{l}\text { Wu et al. } 2014 \\
{[49]}\end{array}$ & $\begin{array}{l}\text { The CHS cohort, } \\
\text { Follow-up, United } \\
\text { States }\end{array}$ & $\mathrm{M}+\mathrm{W}$ & $\begin{array}{l}2792 \text { subjects } \\
\text { including } 362 \text { cases }\end{array}$ & $\geq 65$ & LA in PL & $\begin{array}{l}\text { Q1: } 16.6 \% \\
\text { Q3: } 19.7 \% \\
\text { Q5: } 22.9 \%\end{array}$ & $12.3^{1}$ & $\begin{array}{l}\text { Q2: } 1.00(0.71 ; 1.40) \\
\text { Q3: } 0.90(0.64 ; 1.27) \\
\text { Q4: } 0.88(0.62 ; 1.26) \\
\text { Q5: } 0.88(0.61 ; 1.27)\end{array}$ & $\begin{array}{l}\text { Age; sex; race; enrollment site; } \\
\text { education; smoking status; diabetes; } \\
\text { atrial fibrillation; hypertension; } \\
\text { leisure-time physical activity; BMI; } \\
\text { waist circumference; alcohol; plasma } \\
\text { LC n-3 PUFAs. }\end{array}$ \\
\hline $\begin{array}{l}\text { Daneshmand } \\
\text { et al. } 2016[40]\end{array}$ & $\begin{array}{l}\text { The KIHD cohort; } \\
\text { Follow-up; Finland }\end{array}$ & M & $\begin{array}{l}1828 \text { subjects } \\
\text { including } 153 \text { cases }\end{array}$ & $42-60$ & LA in serum & $\begin{array}{l}\text { Q1: }<23.7 \% \\
\text { Q4: }>29.5 \%\end{array}$ & 21.2 & $\begin{array}{l}\text { Q2: } 0.72(0.46 ; 1.14) \\
\text { Q3: } 0.87(0.55 ; 1.37) \\
\text { Q4: } 1.07(0.68 ; 1.67)\end{array}$ & $\begin{array}{l}\text { Age; examination year; BMI; smoking } \\
\text { status; physical activity; alcohol. }\end{array}$ \\
\hline $\begin{array}{l}\text { Venø et al. } \\
2018[37]\end{array}$ & $\begin{array}{l}\text { The DCH cohort, } \\
\text { Case-cohort, Denmark }\end{array}$ & $\mathrm{M}+\mathrm{W}$ & $\begin{array}{c}55,338 \text { subjects } \\
\text { including } 1755 \text { cases }\end{array}$ & $50-65$ & $\begin{array}{l}\text { LA in adipose } \\
\text { tissue }\end{array}$ & $\begin{array}{l}\text { Q1: }<9.6 \% \\
\text { Q4: }>11.7 \%\end{array}$ & 13.5 & $\begin{array}{l}\text { Q2: } 0.92(0.77 ; 1.09) \\
\text { Q3: } 0.85(0.71 ; 1.02) \\
\text { Q4: } 0.78(0.65 ; 0.93)\end{array}$ & $\begin{array}{c}\text { Age; sex; alcohol; alcohol abstain; } \\
\text { waist circumference adjusted for BMI; } \\
\text { education status; smoking; physical } \\
\text { activity. }\end{array}$ \\
\hline $\begin{array}{l}\text { Marklund et } \\
\text { al. } 2019 \text { [23] }\end{array}$ & $\begin{array}{l}\text { FORCE Consortium; } \\
\text { Individual pooled } \\
\text { analysis of cohort } \\
\text { studies Multinational }\end{array}$ & $\mathrm{M}+\mathrm{W}$ & $\begin{array}{l}\text { The FORCE } \\
\text { Consortium } \\
\text { population including } \\
3705 \text { cases }\end{array}$ & $49-77$ & $\begin{array}{l}\text { LA in PL, TP; } \\
\text { CE, \& AT }\end{array}$ & $\begin{array}{l}\text { PL: } 6.9 \% \\
\text { TP: } 11.4 \% \\
\text { CE: } 11.9 \% \\
\text { RBC: } 4.9 \% \\
\text { AT: } 6.2 \%\end{array}$ & $2.5-31.9$ & $\begin{array}{c}\text { HR per IQR: } \\
\text { PL: } 0.95(0.82 ; 1.10) \\
\text { TP: } 0.84(0.66 ; 1.06) \\
\text { CE } 0.67(0.51 ; 0.88) \\
\text { AT: } 0.87(0.65 ; 1.15) \\
\text { Overall: } 0.88(0.79 ; 0.98)\end{array}$ & $\begin{array}{l}\text { Age; sex; race; field center; BMI; } \\
\text { education; smoking; physical activity; } \\
\text { alcohol; diabetes; treated } \\
\text { hypertension; treated } \\
\text { hypercholesterolemia; aspirin; levels } \\
\text { of ALA, EPA and trans isomers of oleic } \\
\text { acid and trans isomers of linoleic acid. }\end{array}$ \\
\hline
\end{tabular}

Abbreviations: M, men; W, Women; OR, odds ratio; SD, standard deviation; BMI, Body mass index; WHI-OS, Women's Health Initiative Observational Follow-up Study; ARIC, Atherosclerosis Risk in Communities; CE, Cholesterol ester; PL, Phospholipids; HR, Hazard ratio; CI, Confidence interval; Q, Quintile; MORGEN, Monitoring Project on Risk Factors for Chronic Diseases; CHS, Cardiovascular Health Study; LC n-3, Long-chain n-3 polyunsaturated fatty acids; KIHD, Kuopio, Ischaemic Heart Disease Risk Factor Study; DCH, Diet, Cancer and Health; FORCE, Fatty Acid and Outcomes Research Consortium; TP, Total plasma; AT, Adipose tissue; ALA, alpha-linolenic acid; EPA, Eicosapentanoic acid. The presented measures of association was selected for models that included adjustment for lifestyle factors without including dietary covariates whenever possible. The lowest group of exposure was used as reference unless otherwise indicated. ${ }^{1}$ Follow-up time was calculated as person-years of follow-up divided by the total number of participants; ${ }^{2} 99 \%$ confidence interval. 
In summary, limited evidence regarding LA intake and ischemic stroke exist, but LA intake might be associated with a lower risk of total ischemic stroke although the association may depend on macronutrient used to replace LA and the ischemic stroke subtype in question. Several biomarker studies have supported that a high content of LA in blood components or adipose tissue may be associated with a lower risk of total ischemic stroke.

\section{Discussion}

Several prospective follow-up studies have suggested that LC n-3 PUFAs perhaps in particular EPA and DHA may be associated with a lower risk of total ischemic stroke although results have not been consistent. A few studies have suggested that the associations between individual LC n-3 PUFAs may differ amongst subtypes of ischemic stroke with the most beneficial findings observed in ischemic strokes of presumed atherosclerotic origin. Regarding the role of ALA for development of ischemic strokes, most prospective studies have not supported that ALA may be appreciably associated with a lower risk of ischemic stroke. The associations between LA and ischemic stroke have been less studied than the role of n-3 PUFAs, but some biomarker studies have supported that LA might be associated with a lower risk of ischemic stroke. Limited evidence exist regarding LA intake and ischemic stroke, but the association may depend on the macronutrient used to replace LA.

Biomarkers of PUFA exposure may represent useful complementary measures of exposure to investigate the associations with chronic diseases such as ischemic stroke [50]. Dietary studies using estimated PUFA intakes as exposure are limited by measurement error, which may result in loss of statistical power and attenuation of associations toward null. In contrast, the content of PUFAs in blood components or adipose tissue can be assessed more precise and are considered objective biomarkers of exposure [50,51]. Long-term measures of exposure is likely to be of greater etiological relevance in the development of ischemic stroke compared to short-term measures of exposure. Adipose tissue is considered the gold standard due to a slow turnover time possibly reflecting intake of PUFAs during the previous 1-2 years, whereas shorter-term biomarkers such as PUFA content in blood components may represent the exposure up to few months. However, the content of PUFAs in blood components or adipose tissue reflects both intake and metabolism [50]. Also, direct comparison of measures of associations obtained in previous biomarker of PUFAs from different population should be done with caution because differences in PUFA content in human tissue may be largely influenced by differences in the underlying dietary pattern $[50,51]$.

Finally, ischemic stroke is a heterogeneous condition and it is likely that the biological effects of individual LC n-3 PUFAs and LA may differ across subtypes of ischemic stroke of different etiology. Ischemic stroke due to large artery atherosclerosis is considered to be of atherosclerotic origin, whereas ischemic stroke due to small-vessel occlusion may develop as a result of atherosclerosis or lipohyalinosis affecting the smaller penetrating arteries in the brain [52,53]. In contrast, ischemic cardio-embolic strokes are mainly caused by emboli arising from the heart due to arrhythmias, particularly atrial fibrillation or flutter. Furthermore, ischemic stroke may in rare cases develop as a result of nonatherosclerotic vasculopathies or prothrombotic disorders. Thus, these rather distinct potential causes of ischemic stroke underline that separate analyses of ischemic stroke subtypes may contribute with a better understanding of the underlying biology of exposure to PUFAs. Recent results based on data from the DCH cohort [35] and the pooled analyses by Saber et al. [34] indicated that the associations between individual LC n-3 PUFAs and ischemic stroke subtypes may differ. Interestingly, results from the DCH cohort indicated stronger associations for EPA and LA on ischemic stroke due to large artery atherosclerosis than for ischemic stroke due to small-vessel occlusion. This might indicate that potential anti-atherosclerotic properties of marine n-3 PUFAs may be of importance. Anti-atherosclerotic properties of LC n-3 PUFAs may include lowering of triglycerides and perhaps also of atherogenic small-dense LDL particles, a reduction in inflammation and blood pressure, while LA might inhibit the atherosclerotic process by lowering of LDL-cholesterol and blood pressure. EPA seemed to be stronger associated ischemic stroke due to large artery atherosclerosis than DHA in 
the DCH cohort, but whether these differences can be attributed to different biological effects remain unclear, but is not unlikely as many data support that a major effect of DHA on heart disease may be an antiarrhythmic effect.

In addition, the associations may depend on the macronutrients used to replace PUFAs and such substitution aspects together-with focus on dietary patterns-represent interesting areas for future research.

Author Contributions: All authors contributed to the conceptualizations of this manuscript. C.S.B. wrote the first draft of the manuscript and S.K.V. and E.B.S. critically revised the manuscript and contributed with intellectual content. All authors approved the final manuscript.

Funding: This research was funded by The Danish Heart Foundation, grant number 17-R115-A7415-22060, Helene and Georg Jensens and Ethel Merethe and Christian Pontoppidan's Fund. The funding agencies had no role in writing of the manuscript or decision to publish.

Conflicts of Interest: The authors declare no conflicts of interest.

\section{References}

1. Sacco, R.; Kasner, S.; Broderick, J.; Caplan, L.; Connors, J.; Culebras, A.; Elkind, M.; George, M.; Hamdan, A.; Higashida, R.; et al. An Updated Definition of Stroke for the 21st Century. Stroke 2013, 44, 2064-2089. [CrossRef] [PubMed]

2. Hansen, C.; Overvad, K.; Kyrø, C.; Olsen, A.; Tjønneland, A.; Johnsen, S.; Jakobsen, M.; Dahm, C. Adherence to a Healthy Nordic Diet and Risk of Stroke: A Danish Cohort Study. Stroke 2017, 48, 259-264. [CrossRef] [PubMed]

3. Chiuve, S.; Rexrode, K.; Spiegelman, D.; Logroscino, G.; Manson, J.; Rimm, E. Primary prevention of stroke by healthy lifestyle. Circulation 2008, 118, 947-954. [CrossRef] [PubMed]

4. De Caterina, R. N-3 Fatty Acids in Cardiovascular Disease. N. Engl. J. Med. 2011, 364, 2439-2450. [CrossRef] [PubMed]

5. Baker, E.; Miles, E.; Burdge, G.; Yaqoob, P.; Calder, P. Metabolism and functional effects of plant-derived omega-3 fatty acids in humans. Prog. Lipid Res. 2016, 64, 30-56. [CrossRef]

6. Raatz, S.K.; Conrad, Z.; Jahns, L. Trends in linoleic acid intake in the United States adult population: NHANES 1999-2014. Prostaglandins Leukot. Essent. Fatty Acids 2018, 133, 23-28. [CrossRef]

7. Whelan, J.; Fritsche, K. Linoleic acid. Adv. Nutr. 2013, 4, 311-312. [CrossRef]

8. Gebauer, S.; Psota, T.; Harris, W.; Kris-Etherton, P. n-3 Fatty acid dietary recommendations and food sources to achieve essentiality and cardiovascular benefits. Am. J. Clin. Nutr. 2006, 83, 1526-1535. [CrossRef]

9. Bork, C.; Jakobsen, M.; Lundbye-Christensen, S.; Tjønneland, A.; Schmidt, E.; Overvad, K. Dietary intake and adipose tissue content of alpha-linolenic acid and risk of myocardial infarction: A Danish cohort study. Am. J. Clin. Nutr. 2016, 104, 41-48. [CrossRef]

10. Hu, F.; Stampfer, M.; Manson, J.; Rimm, E.; Wolk, A.; Colditz, G.; Hennekens, C.; Willett, W. Dietary intake of alpha-linolenic acid and risk of fatal ischemic heart disease among women. Am. J. Clin. Nutr. 1999, 69, 890-897. [CrossRef]

11. Burdge, G. Is essential fatty acid interconversion an important source of polyunsaturated fatty acids in humans? Br. J. Nutr. 2018, 27, 1-28.

12. Dyerberg, J.; Bang, H.; Stoffersen, E.; Moncada, S.; Vane, J. Eicosapentaenoic acid and prevention of thrombosis and atherosclerosis? Lancet 1978, 2, 117-119. [CrossRef]

13. Dyerberg, J.; Schmidt, E. n-3 fatty acids and cardiovascular disease -observations generated by studies in Greenland Eskimos. Wien. Klin. Wochenschr. 1989, 101, 277-282. [PubMed]

14. Schmidt, E. n-3 fatty acids and the risk of coronary heart disease. Dan. Med. Bull. 1997, 44, 1-22. [PubMed]

15. Calder, P. Marine omega-3 fatty acids and inflammatory processes: Effects, mechanisms and clinical relevance. Biochim. Biophys. Acta 2015, 1851, 469-484. [CrossRef] [PubMed]

16. Mozaffarian, D.; Wu, J. Omega-3 fatty acids and cardiovascular disease: Effects on risk factors, molecular pathways, and clinical events. J. Am. Coll. Cardiol. 2011, 58, 2047-2067. [CrossRef] [PubMed]

17. Saravanan, P.; Davidson, N.; Schmidt, E.; Calder, P. Cardiovascular effects of marine omega-3 fatty acids. Lancet (London, England) 2010, 376, 540-550. [CrossRef] 
18. Rajaram, S. Health benefits of plant-derived alpha-linolenic acid. Am. J. Clin. Nutr. 2014, 100, 443-448. [CrossRef]

19. Wang, D. Dietary n-6 polyunsaturated fatty acids and cardiovascular disease: Epidemiologic evidence. Prostaglandins Leukot. Essent. Fatty Acids 2018, 135, 5-9. [CrossRef]

20. Innes, J.; Calder, P. Omega-6 fatty acids and inflammation. Prostaglandins Leukot. Essent. Fatty Acids 2018, 132, 41-48. [CrossRef]

21. Bork, C.; Baker, E.; Lundbye-Christensen, S.; Miles, E.; Calder, P. Lowering the linoleic acid to alpha-linoleic acid ratio decreases the production of inflammatory mediators by cultured human endothelial cells. Prostaglandins Leukot. Essent. Fatty Acids 2019, 141, 1-8. [CrossRef]

22. Alexander, D.; Miller, P.; Van Elswyk, M.; Kuratko, C.; Bylsma, L. A Meta-Analysis of Randomized Controlled Trials and Prospective Cohort Studies of Eicosapentaenoic and Docosahexaenoic Long-Chain Omega-3 Fatty Acids and Coronary Heart Disease Risk. Mayo Clin. Proc. 2017, 92, 15-29. [CrossRef] [PubMed]

23. Marklund, M.; Wu, J.; Imamura, F.; Del Gobbo, L.; Fretts, A.; de Goede, J.; Shi, P.; Tintle, N.; Wennberg, M.; Aslibekyan, S.; et al. Biomarkers of Dietary Omega-6 Fatty Acids and Incident Cardiovascular Disease and Mortality: An Individual-Level Pooled Analysis of 30 Cohort Studies. Circulation 2019. [CrossRef] [PubMed]

24. Farvid, M.; Ding, M.; Pan, A.; Sun, Q.; Chiuve, S.; Steffen, L.; Willett, W.; Hu, F. Dietary linoleic acid and risk of coronary heart disease: A systematic review and meta-analysis of prospective cohort studies. Circulation 2014, 130, 1568-1578. [CrossRef] [PubMed]

25. De Goede, J.; Verschuren, W.; Boer, J.; Kromhout, D.; Geleijnse, J. Alpha-linolenic acid intake and 10-year incidence of coronary heart disease and stroke in 20,000 middle-aged men and women in the Netherlands. PLoS ONE 2011, 6, e17967. [CrossRef] [PubMed]

26. Fretts, A.; Mozaffarian, D.; Siscovick, D.; Sitlani, C.; Psaty, B.; Rimm, E.; Song, X.; McKnight, B.; Spiegelman, D.; King, I.; et al. Plasma phospholipid and dietary $\alpha$-linolenic acid, mortality, CHD and stroke: The Cardiovascular Health Study. Br. J. Nutr. 2014, 112, 1206-2013. [CrossRef] [PubMed]

27. Rhee, J.; Kim, E.; Buring, J.; Kurth, T. Fish Consumption, Omega-3 Fatty Acids, and Risk of Cardiovascular Disease. Am. J. Prev. Med. 2017, 52, 10-19. [CrossRef] [PubMed]

28. Larsson, S.; Virtamo, J.; Wolk, A. Dietary fats and dietary cholesterol and risk of stroke in women. Atherosclerosis 2012, 221, 282-286. [CrossRef] [PubMed]

29. Bork, C.; Venø, S.; Lundbye-Christensen, S.; Jakobsen, M.; Tjønneland, A.; Schmidt, E.; Overvad, K. Dietary Intake of $\alpha$-Linolenic Acid Is Not Appreciably Associated with Risk of Ischemic Stroke among Middle-Aged Danish Men and Women. J. Nutr. 2018, 148, 952-958. [CrossRef]

30. Iso, H.; Rexrode, K.; Stampfer, M.; Manson, J.; Colditz, G.; Speizer, F.; Hennekens, C.; Willett, W. Intake of fish and omega-3 fatty acids and risk of stroke in women. JAMA 2001, 285, 304-312. [CrossRef]

31. Iso, H.; Sato, S.; Umemura, U.; Kudo, M.; Koike, K.; Kitamura, A.; Imano, H.; Okamura, T.; Naito, Y.; Shimamoto, T. Linoleic acid, other fatty acids, and the risk of stroke. Stroke 2002, 33, 2086-2093. [CrossRef] [PubMed]

32. Yaemsiri, S.; Sen, S.; Tinker, L.; Robinson, W.; Evans, R.; Rosamond, W.; Wasserthiel-Smoller, S.; He, K. Serum fatty acids and incidence of ischemic stroke among postmenopausal women. Stroke 2013, 44, 2710-2717. [CrossRef] [PubMed]

33. Bork, C.; Venø, S.; Lundbye-christensen, S.; Jakobsen, M.; Tjønneland, A.; Calder, P.; Overvad, K.; Schmidt, E. Adipose tissue content of alpha-linolenic acid and the risk of ischemic stroke and ischemic stroke subtypes: A Danish case-cohort study. PLoS ONE 2018, 13, e0198927. [CrossRef] [PubMed]

34. Saber, H.; Yakoob, M.; Shi, P.; Longstreth, W.; Lemaitre, R.; Siscovick, D.; Rexrode, K.; Willett, W.; Mozaffarian, D. Omega-3 Fatty Acids and Incident Ischemic Stroke and Its Atherothrombotic and Cardioembolic Subtypes in 3 US Cohorts. Stroke 2017, 48, 2678-2685. [CrossRef] [PubMed]

35. Venø, S.; Bork, C.; Jakobsen, M.; Lundbye-Christensen, S.; McLennan, P.; Bach, F.; Overvad, K.; Schmidt, E. Marine n-3 Polyunsaturated Fatty Acids and the Risk of Ischemic Stroke. Stroke 2019, 50, 274-282. [CrossRef] [PubMed]

36. Venø, S.; Schmidt, E.; Jakobsen, M.; Lundbye-Christensen, S.; Bach, F.; Overvad, K. Substitution of Linoleic Acid for Other Macronutrients and the Risk of Ischemic Stroke. Stroke 2017, 48, 3190-3195. [CrossRef] [PubMed] 
37. Venø, S.; Bork, C.; Jakobsen, M.; Lundbye-Christensen, S.; Bach, F.; Overvad, K.; Schmidt, E. Linoleic Acid in Adipose Tissue and Development of Ischemic Stroke: A Danish Case-Cohort Study. J. Am. Heart Assoc. 2018, 7, e009820. [CrossRef]

38. De Goede, J.; Verschuren, W.; Boer, J.; Kromhout, D.; Geleijnse, J. N-6 and n-3 fatty acid cholesteryl esters in relation to incident stroke in a Dutch adult population: A nested case-control study. Nutr. Metab. Cardiovasc. Dis. 2013, 23, 737-743. [CrossRef]

39. Yamagishi, K.; Folsom, A.; Steffen, L. Plasma fatty acid composition and incident ischemic stroke in middle-aged adults: The Atherosclerosis Risk in Communities (ARIC) Study. Cerebrovasc. Dis. 2013, 36, 38-46. [CrossRef]

40. Daneshmand, R.; Kurl, S.; Tuomainen, T.; Virtanen, J. Associations of serum n-3 and n-6 PUFA and hair mercury with the risk of incident stroke in men: The Kuopio Ischaemic Heart Disease Risk Factor Study (KIHD). Br. J. Nutr. 2016, 115, 1851-1859. [CrossRef]

41. He, K.; Rimm, E.; Merchant, A.; Rosner, B.; Stampfer, M.; Willett, W.; Ascherio, A. Fish Consumption and Risk of Stroke in Men. JAMA 2002, 288, 3130-3136. [CrossRef] [PubMed]

42. De Goede, J.; Verschuren, W.; Boer, J.M.; Kromhout, D.; Geleijnse, J. Gender-specific associations of marine n-3 fatty acids and fish consumption with 10-year incidence of stroke. PLoS ONE 2012, 7, e33866. [CrossRef] [PubMed]

43. Montonen, J.; Järvinen, R.; Reunanen, A.; Knekt, P. Fish consumption and the incidence of cerebrovascular disease. Br. J. Nutr. 2009, 102, 750-756. [CrossRef] [PubMed]

44. Kippler, M.; Larsson, S.; Berglund, M.; Glynn, A.; Wolk, A.; Åkesson, A. Associations of dietary polychlorinated biphenyls and long-chain omega-3 fatty acids with stroke risk. Environ. Int. 2016, 94, 706-711. [CrossRef] [PubMed]

45. Wallström, P.; Sonestedt, E.; Hlebowicz, J.; Ericson, U.; Drake, I.; Persson, M.; Gullberg, B.; Hedblad, B.; Wirfält, E. Dietary fiber and saturated fat intake associations with cardiovascular disease differ by sex in the Malmö Diet and Cancer Cohort: A prospective study. PLoS ONE 2012, 7, e31637. [CrossRef]

46. Yamagishi, K.; Iso, H.; Date, C.; Fukui, M.; Wakai, K.; Kikuchi, S.; Inaba, Y.; Tanabe, N.; Tamakoshi, A. Fish, omega-3 polyunsaturated fatty acids, and mortality from cardiovascular diseases in a nationwide community-based cohort of Japanese men and women the JACC (Japan Collaborative Cohort Study for Evaluation of Cancer Risk) Study. J. Am. Coll. Cardiol. 2008, 52, 988-996. [CrossRef] [PubMed]

47. Bork, C.; Venø, S.; Lasota, A.; Lundbye-Christensen, S.; Schmidt, E. Marine and plant-based n-3 PUFA and atherosclerotic cardiovascular disease. Proc. Nutr. Soc. 2019. [CrossRef]

48. Mozaffarian, D.; Lemaitre, R.; King, I.; Song, X.; Huang, H.; Sacks, F.; Rimm, E.; Wang, M.; Siscovick, D. Plasma phospholipid long-chain $\omega-3$ fatty acids and total and cause-specific mortality in older adults: A cohort study. Ann. Intern. Med. 2013, 158, 515-525. [CrossRef]

49. Wu, J.; Lemaitre, R.; King, I.; Song, X.; Psaty, B.; Siscovick, D.; Mozaffarian, D. Circulating omega-6 polyunsaturated fatty acids and total and cause-specific mortality: The Cardiovascular Health Study. Circulation 2014, 130, 1245-1253. [CrossRef]

50. Hodson, L.; Skeaff, C.; Fielding, B. Fatty acid composition of adipose tissue and blood in humans and its use as a biomarker of dietary intake. Prog. Lipid Res. 2008, 47, 348-380. [CrossRef]

51. Arab, L.; Akbar, J. Biomarkers and the measurement of fatty acids. Public Health Nutr. 2002, 5, 865-871. [CrossRef] [PubMed]

52. Adams, H.J.; Bendixen, B.; Kappelle, L.; Biller, J.; Love, B.; Gordon, D.; Marsh, E. Classification of subtype of acute ischemic stroke. Definitions for use in a multicenter clinical trial. TOAST. Trial of Org 10172 in Acute Stroke Treatment. Stroke 1993, 24, 35-41. [CrossRef] [PubMed]

53. Lammie, G. Pathology of small vessel stroke. Br. Med. Bull. 2000, 56, 296-306. [CrossRef] [PubMed]

(C) 2019 by the authors. Licensee MDPI, Basel, Switzerland. This article is an open access article distributed under the terms and conditions of the Creative Commons Attribution (CC BY) license (http://creativecommons.org/licenses/by/4.0/). 